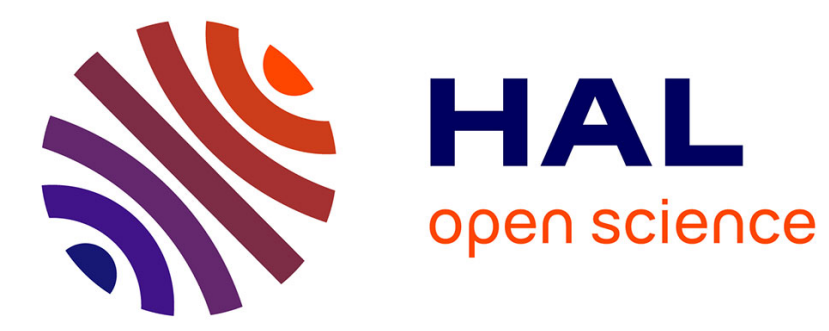

\title{
Non-specific interactions govern cytosolic diffusion of nanosized objects in mammalian cells
}

Fred Etoc, Elie Balloul, Chiara Vicario, Davide Normanno, Domenik Lisse, Assa Sittner, Jacob Piehler, Maxime Dahan, Mathieu Coppey

\section{- To cite this version:}

Fred Etoc, Elie Balloul, Chiara Vicario, Davide Normanno, Domenik Lisse, et al.. Non-specific interactions govern cytosolic diffusion of nanosized objects in mammalian cells. Nature Materials, 2018, 17 (8), pp.740-746. 10.1038/s41563-018-0120-7 . hal-01871099

\section{HAL Id: hal-01871099 \\ https://hal.sorbonne-universite.fr/hal-01871099}

Submitted on 10 Sep 2018

HAL is a multi-disciplinary open access archive for the deposit and dissemination of scientific research documents, whether they are published or not. The documents may come from teaching and research institutions in France or abroad, or from public or private research centers.
L'archive ouverte pluridisciplinaire HAL, est destinée au dépôt et à la diffusion de documents scientifiques de niveau recherche, publiés ou non, émanant des établissements d'enseignement et de recherche français ou étrangers, des laboratoires publics ou privés. 


\title{
1 Non-specific interactions govern cytosolic diffusion of nano-sized 2 objects in mammalian cells
}

3

4

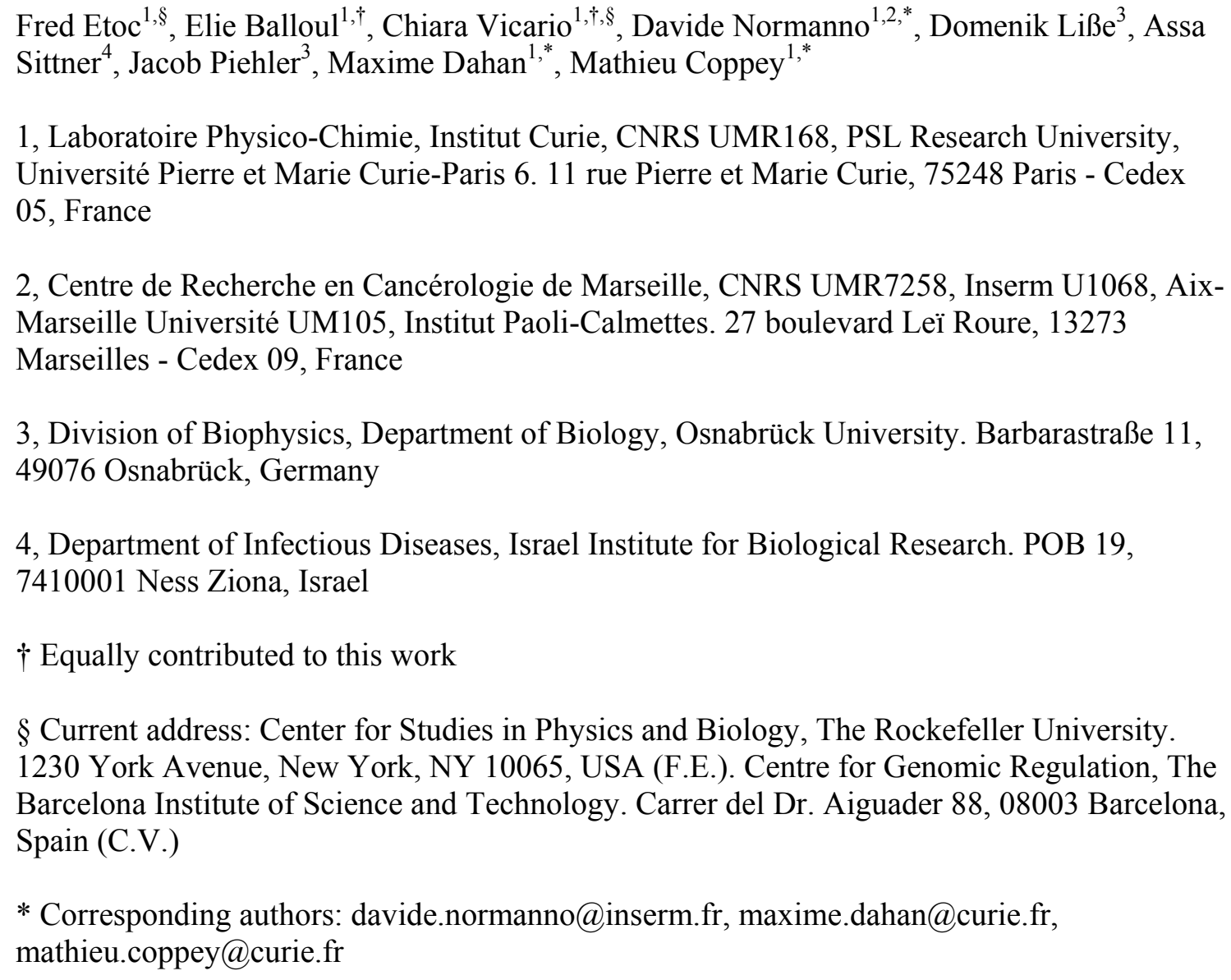

\begin{abstract}
The diffusivity of macromolecules in the cytoplasm of eukaryotic cells varies over orders of magnitude and dictates the kinetics of cellular processes. However, a general description associating the Brownian or anomalous nature of intracellular diffusion to the architectural and biochemical properties of the cytoplasm has not been achieved. Here, we measure the mobility of individual fluorescent nanoparticles in living mammalian cells to obtain a comprehensive analysis of cytoplasmic diffusion. We identify a correlation between tracer size, its biochemical nature, and its mobility. Inert particles with size equal or below $50 \mathrm{~nm}$ behave as Brownian particles diffusing in a medium of low viscosity with negligible effects of molecular crowding. Increasing the strength of non-specific interactions of the nanoparticles within the cytoplasm gradually reduces their mobility and leads to sub-diffusive behaviour. These experimental observations and the transition from Brownian to sub-diffusive motion can be recapitulated in a minimal phenomenological model.
\end{abstract}


Cytoplasmic diffusion plays a fundamental role in many cellular processes. For instance, signalling pathways are activated by chemical species that diffuse and react in the microenvironment of the cytoplasm, therefore molecular diffusion dictates the dynamics of cellular responses ${ }^{1}$, such as MAPK activation ${ }^{2}$ or the kinetics of search processes ${ }^{3}$. Assessing the physical nature of the cytoplasm, and its consequences on the mobility of biomolecules, is essential for understanding, and eventually controlling, biochemical processes in living cells.

The cytoplasm of eukaryotic cells is generally described as a bi-phasic poro-elastic medium $^{4,5}$, composed of a fluid phase, the cytosol, consisting of water and soluble proteins, and a solid phase consisting of the cytoskeleton, endomembranes, organelles, and supramolecular complexes ${ }^{6}$. Starting from the seminal work on the mobility of IgG and BSA ${ }^{7}$, a plethora of -often conflicting- findings about cytosolic diffusion has been reported in the literature (for recent reviews see Ref. 8,9). Early fluorescence recovery after photo-bleaching (FRAP) experiments with inert Dextran and Ficoll pointed to a cytosol slightly (1 to 4 times) more viscous than water ${ }^{10,11}$, while experiments with various proteins, as reviewed in Ref. 6 , yielded to an apparent cytosol viscosity varying between 0.007 (for Insulin ${ }^{12}$ ) and 0.49 (for $\left.\mathrm{GFP}^{13}\right)$. Theoretical work ${ }^{14}$ as well as in vitro experiments in dense, crowded solutions and in cell extracts ${ }^{15,16}$ have also suggested that diffusion in the cytoplasm could deviate from Brownian motion. Experiments in cells have led to the observation of both phenomena: Brownian diffusion ${ }^{17,18}$ and sub-diffusive motion ${ }^{19-21}$. Since all these results have been obtained with a variety of tracers and in different experimental conditions, a fundamental, long-lasting ${ }^{22}$ and yet unresolved question is whether a unified framework can account for the large variability of behaviours observed for cytosolic diffusion.

Two major classes of phenomena can affect the mobility of a tracer and potentially lead to sub-diffusion. First, obstacles are expected to induce steric hindrance, limiting the accessible space, and thereby slow down diffusion ${ }^{15,23}$. We refer to these geometric effects as "obstructive molecular crowding" caused by a high concentration of macromolecules in the cytoplasm $^{18,24}$. Second, biochemical (e.g. electrostatic or hydrophobic) interactions with elements of the cytoplasm, referred here as "non-specific interactions", can give rise to transient immobilization and reduced mobility. In the present work, using single-particletracking ${ }^{25}$ of fluorescent nanoparticle (NP) tracers, we show that inert objects, with size below $\sim 75 \mathrm{~nm}$, are not affected by obstructive molecular crowding and behave as Brownian particles diffusing in a medium of low viscosity. For tracers with given size $(\sim 25 \mathrm{~nm})$ but with increasing levels of non-specific interactions, we find that the effective diffusivity is progressively slowed down by more than 3 orders of magnitude while diffusion gradually becomes anomalous. We show that a simple continuous time random walk model, in which the strength of non-specific interactions is captured into a single parameter, is sufficient to recapitulate the intracellular mobility of all the different probes analysed.

\section{Tracers of similar size have different diffusivities}

To systematically inspect the cytoplasmic mobility of nano-sized objects, we first established a methodology to quantify and compare the diffusivity of two different NPs with similar size: rhodamine-doped, streptavidin-coated polystyrene/polymethacrylate particles (called " $25 \mathrm{~nm}$ Rho-NPs", but of measured diameter 35nm \pm 8nm) and Amino (PEG) 655 ITK TM Quantum Dots (called "QDs", about 20nm in diameter ${ }^{26}$ ). Probes were internalized in HeLa cells through pinocytic loading ${ }^{27}$, which allows the delivery of 20 to 50 individual NPs per cell. Control experiments ensured that pinocytic loading effectively yielded dispersed NPs in the 
cytosol of viable, living cells (see Methods, Supplementary Fig. 1-7, and Supplementary Video 1-5).

Cytoplasmic diffusion of individual NPs was recorded by time-lapse microscopy and quantified using single-particle-tracking analysis. From each individual trajectory, we extracted two complementary parameters. First, we estimated the mobility at short time scales computing the time-averaged Mean Square Displacement (taMSD) at $60 \mathrm{~ms},\left\langle r_{60 \mathrm{~ms}}^{2}\right\rangle_{\mathrm{T}}$ (see Methods). For convenience, we express this observable in terms of a diffusion coefficient $D_{60 \mathrm{~ms}}=\left\langle r_{60 \mathrm{~ms}}^{2}\right\rangle_{\mathrm{T}} / 4 \delta$, (with $\delta=60 \mathrm{~ms}$ ). Second, we characterized the mobility at long time scales computing the anomalous exponent. We selected trajectories longer than 40 time points and fitted each individual taMSD curve with a general model of diffusion $\left\langle r^{2}\right\rangle_{\mathrm{T}} \sim t^{\alpha}$, from which we determined the anomalous exponent $\alpha$ as a single fitting parameter. To represent the behaviour of the whole population of NPs in each condition, the distribution of the parameters $\alpha$ and $D_{60 \mathrm{~ms}}$ arising from each individual trajectory was represented as a twodimensional density $\operatorname{map}^{28}$ (Fig 1). Given the robustness with respect to the choice of parameters used for their computation (Supplementary Fig. 8), $\alpha-D_{60 \mathrm{~ms}}$ maps can be used as benchmark to systemically compare the mobility of nano-sized objects in the cytoplasm of living cells.

In the case of $25 \mathrm{~nm}$ Rho-NPs (Fig. 1a,b), we found a predominant sub-population with $D_{60 \mathrm{~ms}}$ peaking at $1.34 \pm 0.07 \mu \mathrm{m}^{2} \cdot \mathrm{s}^{-1}$ (all values are mean \pm standard error of the mean (SEM), the number $N$ of trajectories considered in the analysis is reported in figure captions) and $\alpha=0.92 \pm 0.01$, close to $0.96 \pm 0.02$, the value obtained for simulated Brownian particles (see Methods). In addition to (fast) Brownian particles, $25 \mathrm{~nm}$ Rho-NPs presented a second (slow) population $(\sim 30 \%)$ with reduced instantaneous mobility, $D_{60 \mathrm{~ms}}=0.51 \pm$ $0.05 \mu \mathrm{m}^{2} \cdot \mathrm{s}^{-1}$, and strongly sub-diffusive $\alpha=0.18 \pm 0.02$ (see Supplementary Fig. 9 and Methods).

For QDs, we found a single, broad peak in the $\alpha-D_{60 \mathrm{~ms}}$ plane (Fig. 1c,d). The distribution of $D_{60 \mathrm{~ms}}$ spanned more than three orders of magnitude, as for $25 \mathrm{~nm}$ Rho-NPs, but peaked at $0.15 \pm 0.01 \mu \mathrm{m}^{2} \cdot \mathrm{s}^{-1}$, one order of magnitude smaller than for $25 \mathrm{~nm}$ Rho-NPs. Furthermore, the exponent $\alpha=0.71 \pm 0.01$ pointed to a deviation from Brownian diffusion. As reference, the distribution obtained with the same QDs in a glycerol solution $(80 \% \mathrm{v} / \mathrm{v})$ is more homogeneous (Fig. 1e), with $\alpha \sim 1$, indicative of Brownian diffusion (Fig. 1f). Thus, the heterogeneity in QDs mobility, its deviation from Brownian diffusion and from the behaviour of $25 \mathrm{~nm}$ Rho-NPs does not arise from a broad size distribution of QDs nor from specific features of single-particle-tracking analysis but is intrinsic to the intracellular mobility of QDs.

\section{Rho-NPs undergo Brownian motion for sizes up to 50nm}

We next investigated the range of particle sizes over which the observation of Brownian motion was valid by comparing the mobility of Rho-NPs of increasing dimension $(15,25,50$ and $75 \mathrm{~nm}$ in diameter, see Supplementary Fig. 10). 75nm Rho-NPs were microinjected since pinocytic loading does not work for large objects. In the $\alpha-D_{60 \mathrm{~ms}}$ plane, NPs below or equal to $50 \mathrm{~nm}$ had similar features, majorly peaking at $\alpha \sim 1$ and with $D_{60 \mathrm{~ms}}$ on the order of $1 \mu \mathrm{m}^{2} \cdot \mathrm{s}^{-1}$ (Fig. 2a-c). Remarkably, $D_{60 \mathrm{~ms}}$ decreased as the inverse of NPs size, as expected for diffusion in a homogeneous medium, and the apparent cytoplasmic viscosity -estimated using the Stokes-Einstein equation- was 10-15 times higher than that of water (Supplementary Table 
1), i.e. about five times higher than previous reports for the viscosity of the fluid phase of the cytoplasm $^{29,30}$.

The diffusive properties of $75 \mathrm{~nm}$ Rho-NPs were strikingly different (Fig. 2d). The average $D_{60 \mathrm{~ms}}$ was $0.22 \pm 0.03 \mu \mathrm{m}^{2} \cdot \mathrm{s}^{-1}$ (with a median value of $0.015 \mu \mathrm{m}^{2} \cdot \mathrm{s}^{-1}$ ), much smaller than the three other samples. Furthermore, trajectories were strongly sub-diffusive, with low $\alpha$ values $(0.56 \pm 0.03)$. We concluded that there is a clear cut-off, between 50 and $75 \mathrm{~nm}$, in the size dependence of NPs mobility.

156

157

158

159

160

161

\section{QDs show sub-diffusive behaviour}

To assess the departure of QDs from Brownian diffusion, we computed the ensemble average of all individual taMSDs, $\left\langle\left\langle r^{2}\right\rangle_{\mathrm{T}}\right\rangle_{\mathrm{E}}$. Although $\left\langle\left\langle r^{2}\right\rangle_{\mathrm{T}}\right\rangle_{\mathrm{E}}$ grew sub-linearly (Fig. 3a and Supplementary Fig. 11), a single exponent $\alpha$ failed to describe the experimental data since it evolved from 1 at short time scales (tens of milliseconds) down to 0.7 at time scales on the order of seconds. We found that this variation of $\alpha$ was due to the ensemble averaging procedure, which samples trajectories unevenly. Diffusing NPs can escape the volume of observation, determined by the depth of field of the microscope $(\sim 500 \mathrm{~nm})$, resulting in an exponential distribution of trajectory lengths (mean duration of 1.4s, Supplementary Fig. 12). Due to the limited imaging depth, particles with small $D_{60 \mathrm{~ms}}$ tend to stay longer within the observation volume and, thus, yield longer trajectories than fast diffusing particles ${ }^{31}$. Therefore, averaging together all individual taMSDs led to a progressive decrease with time of the slope of the ensemble average of taMSDs, as the contribution of slow NPs becomes predominant at longer times (Supplementary Fig. 13).

To overcome this bias, we considered trajectory datasets homogeneous in length. Specifically, we chose trajectories lasting at least $N$ steps to build the ensemble average of taMSDs, $\left\langle\left\langle r^{2}\right\rangle_{\mathrm{T} \geq \mathrm{N} \Delta \mathrm{t}}\right\rangle_{\mathrm{E}}$, which we computed up to time $N \cdot \Delta t$. By doing so, we ensured that all trajectories contributed equally to the ensemble average over the whole time span considered. In Fig. $3 b,\left\langle\left\langle r^{2}\right\rangle_{\mathrm{T} \geq \mathrm{N} \Delta \mathrm{t}}\right\rangle_{\mathrm{E}}$ is plotted in log-log scale for $N$ equal to 30 , 75 and 150 steps (corresponding, respectively, to a time window of $0.9,2.25$, and 4.5s). Strikingly, even if the three MSD curves include varying partitioning of the different QDs behaviours (the longer the trajectories are, the slower the mobility is), all curves are linear in log-log scale with an identical exponent $\alpha \sim 0.82$, indicative of a uniform behaviour. We thus concluded that QDs diffusion can be well described by a homogeneous sub-diffusive process.

\section{QDs sub-diffusion arises from non-specific interactions}

We first inspected if confinement by the cytoskeleton or internal membranes could explain the observed QDs sub-diffusion. We used different drugs known to disrupt the actin meshwork, the microtubule network, and the Golgi apparatus (Latrunculin-A, Nocodazole, and Brefeldin-A, respectively), but we found no substantial change in the sub-diffusive behaviour of QDs (Supplementary Fig. 14). We also analysed QD trajectories using a confined diffusion model to fit individual $\left\langle r^{2}\right\rangle_{\mathrm{T}}$ curves. We found no strong evidence for confinement with and without drugs (Supplementary Fig. 15 and Supplementary Table 2). We concluded that, for NPs below the critical cut-off size of $75 \mathrm{~nm}$, geometric restrictions due to confinement within the cytoskeleton meshwork or the Golgi have no pronounced effects on cytoplasmic mobility. 
We next aimed at discriminating between steric hindrance due to high density of obstacles and transient non-specific interactions. In the latter case, anomalous diffusion would arise from a broad distribution of interaction times, whose specific signature would be the loss of equivalence between temporal and ensemble averages, a phenomenon called ergodicity breaking $^{32}$. We therefore performed a simple statistical test for ergodicity ${ }^{33}$. Following the approach proposed by Weigel et $a .^{34}$, we compared the distribution of time-averaged square displacements (shown in Fig. 1c) to the distribution of ensemble-averaged square displacements. As visible in Fig. 3c, the distribution of time-averaged square displacements is much broader that the distribution of ensemble-averaged square displacements, an indication of ergodicity breaking (observed also under Brefeldin-A and Latrunculin-A treatments, see Supplementary Fig. 16a). In contrast, QDs diffusing in glycerol satisfied ergodicity (Fig. 3d). We also observed aging in the trajectories ${ }^{34,35}$ (Supplementary Fig. 16b), an additional signature of ergodicity breaking. Altogether, our analysis strongly supports the view that nonspecific interactions rather than obstructive molecular crowding are at the origin of subdiffusion.

To further demonstrate the role of non-specific interactions, we hypothesized that diffusion should be affected by the surface properties of the tracer and we thus screened QDs with different surface chemistries and charges. We found the distribution of $D_{60 \mathrm{~ms}}$ to strongly depend on the surface coating of QDs (Fig. 3e) but with no correlation between the electrostatic potential and the degree of reduced mobility (Supplementary Table 3). Although the passivation of QDs surface greatly increased mobility, neither PEG nor streptavidin coating resulted in QDs as diffusive as Rho-NPs. Most likely, the strongly interacting QDs core still contributed to non-specific binding even after passivation.

\section{A minimal CTRW model recapitulates the diffusion of all NPs}

As shown, the cytoplasmic mobility of particles of similar size $(\sim 25 \mathrm{~nm})$ varied over several orders of magnitude, with median instantaneous diffusivity coefficients $D_{60 \mathrm{~ms}}$ ranging from $0.005 \mu \mathrm{m}^{2} \cdot \mathrm{s}^{-1}$, for QDs without any additional surface coating (QDs-Itk-COOH), up to $1 \mu \mathrm{m}^{2} \cdot \mathrm{s}^{-1}$ for Rho-NPs. Additionally, we observed both Brownian and anomalous diffusion. We thus wondered if this large variability of cytoplasmic mobility could be recapitulated in a simple, unified framework. First, we supposed the diffusion coefficient of a perfectly inert 25 $\mathrm{nm}$ object to be $10 \mu \mathrm{m}^{2} \cdot \mathrm{s}^{-1}$, a value corresponding to the fastest intracellular diffusion coefficient reported in the literature for objects (dextran polymers ${ }^{29}$ ) of that size. We next hypothesized that non-specific interactions would affect the diffusive properties of NPs through transient binding to immobile or slowly moving objects. Specifically, we considered a continuous time random walk (CTRW) model with a waiting (immobilization) time distribution, $P(t)$, between each step of the walk, of the form: $P(t)=\gamma \cdot t_{0}^{\gamma} / t^{1+\gamma}$ (defined for $\left.t>t_{0}\right)$. The lower bound $t_{0}$ of the distribution is taken here to be the acquisition time and $\gamma$ is a positive parameter, encompassing the strength of non-specific interactions. The mean waiting time $\langle t\rangle=\int_{\mathrm{t}_{0}}^{\infty} t P(t) d t$ is equal to $\gamma t_{0} /(\gamma-1)$ for $\gamma>1$ and diverges for $0<\gamma \leq 1$. For large $\gamma$, the mean immobilisation time is close to $t_{0}$ and it increases for decreasing $\gamma$ values.

We simulated trajectories using our minimal CTRW model for different values of $\gamma$ using conditions similar to our experiments (see Methods). Remarkably, the simulated trajectories recapitulated all the different behaviours observed. For very large $\gamma$ (i.e. $\langle t\rangle \approx t_{0}$ ), simulated trajectories showed free diffusion $(\alpha=0.97 \pm 0.01)$ and $D_{60 \mathrm{~ms}}=9.8 \pm 0.1 \mu \mathrm{m}^{2} \cdot \mathrm{s}^{-1}$, thus 
very close to the value assumed for perfectly inert tracers (Fig. $4, \gamma=\infty$ ). As $\gamma$ decreases toward 1, diffusion remained Brownian $(\alpha \sim 1)$ but with reduced instantaneous diffusivity coefficients $D_{60 \mathrm{~ms}}$, due to the increasing mean immobilisation time (Fig. $4, \gamma=10$ to 1 ). This regime recapitulated the diffusive properties of Rho-NPs. Interestingly, when $\gamma$ was lower than 1 (Fig. 4, $\gamma=0.5$ ), the mean immobilisation time $\langle t\rangle$ diverged, the distribution in the $\alpha$ $D_{60 \mathrm{~ms}}$ plane became elongated with a positive correlation as observed experimentally for QDs (Fig. 1d). Finally, for very small values of $\gamma$ (Fig. 4, $\gamma=0.1$ ), because of the finite observation time, trajectories could only probe a limited number of immobilisation times. This resulted in a large variability in the mobility of individual objects and in a distribution in the $\alpha-D_{60 \mathrm{~ms}}$ plane that recapitulated the feature observed for "naked" QDs (QDs-Itk-COOH).

Given the ability of our model to recapitulate the diffusivity of various NPs, we wondered if we could find a perfectly inert NP in the same size range for which we should observe Brownian diffusion with a diffusion coefficient close to $10 \mu \mathrm{m}^{2} \cdot \mathrm{s}^{-1}$. To this end, we probed the mobility of ferritin NPs passivated with polyethylene glycols (PEGs), which have a size of about $25 \mathrm{~nm}$, thus similar to that of the other NPs analysed, and which were recently shown to behave as highly inert probes $^{36}$. Remarkably, we observed a fast diffusion of ferritin NPs (Fig. 5, top-left panel) with a mean $D_{60 \mathrm{~ms}}=14.2 \pm 0.95 \mu \mathrm{m}^{2} \cdot \mathrm{s}^{-1}\left(\right.$ median $\left.D_{60 \mathrm{~ms}}=6.8 \mu \mathrm{m}^{2} \cdot \mathrm{s}^{-1}\right)$, about 10 times larger than for $25 \mathrm{~nm}$ Rho-NPs and very close to the value expected for an intracellular viscosity between 2 to 4 times that of water. The exponent $\alpha$ was $0.86 \pm 0.01$ lower than expected for purely Brownian particles, probably because of the broad distribution of MSDs due to rapid (out-of-plane) motion of ferritin NPs and the consequent short exposure time and fast frame rate required to track them.

Overall, our simple phenomenological model based on transient immobilisation is capable of recapitulating all the diffusive properties observed in HeLa cells for the different NPs analysed. The model also provides a ranking of NPs mobility according to the strength of their non-specific interactions (Fig. 5, left panels). Remarkably, the ordered $\alpha-D_{60 \mathrm{~ms}}$ maps show a very similar trend for a wide range of adherent mammalian cells, including primary cells (Fig. 5). These results support the notion that the transport properties of NPs of similar size are set by the physical nature of the cell interior and by the NPs surface properties rather than by specific features of different cell types. Altogether, $\alpha-D_{60 \mathrm{~ms}}$ maps constitute a gauge to assess the degree of non-specific interactions of NPs within the cytoplasm.

\section{Addressing the cytosolic diffusion of nano-sized objects}

Two major frameworks have long been competing to explain sub-diffusion in the cytosol, one based on geometrical constraints ${ }^{37}$ and one on biochemical interactions ${ }^{38}$. For objects below the "pore" size of the cytoplasm (defined as the interstitial size of the cytoplasm "solid" matrix), our results unequivocally point to the predominant role of biochemical interactions. The first and most compelling argument comes from the fact that if mobility would be hindered by geometric obstructions all particles of the same physical size should be affected, irrespective of their composition and surface properties. On the contrary, we found very heterogeneous diffusive dynamics, including Brownian particles diffusing as fast as $14 \mu \mathrm{m}^{2} \cdot \mathrm{s}^{-1}$. Thus, not only proteins ${ }^{39}$ but also cytoplasmic objects with size of a few tens of nanometres can diffuse freely in a fluid phase barely more viscous than water. The second argument, based on the detailed analysis of QD trajectories, comes from the observed ergodicity breaking which dismisses the geometrical argument in favour of the biochemical hypothesis. 
Yet, for NPs showing pronounced non-specific interactions, as for instance QDs-Itk-COOH, one could wonder whether the reduced mobility might be caused by an increased effective NPs size, due to a stable protein corona forming around them ${ }^{40}$. However, as shown by RhoNPs data, the change in diffusivity between NPs of 15 and $50 \mathrm{~nm}$ is only a factor 2 . Thus, a putative corona effect cannot account for the spread over more than three orders of magnitude observed for different types of NPs. Concerning the sub-diffusive behaviour of QDs, given that time-averaged MSD curves have exponents smaller than one (mean $\alpha=0.82$, Supplementary Fig. 17), a subordinated diffusion process ${ }^{41}$, involving both ergodic viscoelastic effects and non-ergodic immobilizations, could be invoked. Yet, if cytoplasm would behave as a viscoelastic medium for probes of $25 \mathrm{~nm}$ in size, we should have observed sub-diffusion for all the NPs employed. However, given that Rho-NPs showed Brownian diffusion, we can exclude a role for cytoplasmic viscoelasticity at this particular scale.

In summary, we propose a unified framework to describe the diffusive dynamics of nanosized objects in the cytoplasm of eukaryotic cells (Fig. 6). First, as verified in HeLa cells and in line with previous reports ${ }^{10,11,42}$, diffusion is unconfined as long as the object size does not exceed the pore size of the cytoplasm (between 50 and $75 \mathrm{~nm}$ ). Above this size, diffusion is restricted by the solid meshwork of the cell and movements of the probes, happening on timescale of minutes, are mainly dictated by active stirring ${ }^{43}$ and cytoskeleton dynamics ${ }^{44,45}$. Second, the intrinsic viscosity of the cytosolic fluid phase is low ( 2 to 4 time the viscosity of water), confirming previous results ${ }^{29,30}$. Third, diffusion can be slowed down by non-specific interactions with intracellular components causing an increase of the apparent viscosity. If the strength of interactions is weak, diffusion remains Brownian, whereas sub-diffusive behaviour arises when the distribution of immobilisation times is broad enough so that the average immobilisation time diverges. The nature of the components to which tracers bind is presumably diverse; nevertheless, they have to be sufficiently immobile to considerably slow down the diffusion of the interacting probes. These intracellular "binders" can be organelles, vesicles, internal membranes, as well as macromolecular complexes bigger than the pore size, thus undergoing constrained motion in the cytoplasm.

Importantly for further methodological developments, our tracking assay and the $\alpha-D_{60 \mathrm{~ms}}$ representation provide a quantitative benchmarking tool for the design of perfectly inert NPs. The assay can be used to systematic screen the surface properties of NPs (such as the charge or the nature of the surface passivation coating). In particular, our findings indicate that the design of functionalization strategies for nanoparticle delivery ${ }^{46}$ and manipulation ${ }^{47}$ in the cytoplasm, an important goal in nano-biosciences, should not be limited to testing endosomal escape and cytosolic localization. It should also include the analysis of cytoplasmic mobility of NPs, as it can dramatically vary and affect the ability of NPs to reach molecular targets inside cells.

\section{References}

1. Bénichou, O., Chevalier, C., Klafter, J., Meyer, B. \& Voituriez, R. Geometry-controlled kinetics. Nat. Chem. 2, 472-477 (2010).

2. Hellmann, M., Heermann, D. W. \& Weiss, M. Enhancing phosphorylation cascades by anomalous diffusion. Europhys. Lett. 97, 58004 (2012).

3. Izeddin, I. et al. Single-molecule tracking in live cells reveals distinct target-search strategies of transcription factors in the nucleus. eLife 3, e02230 (2014). 
4. Hou, L., Lanni, F. \& Luby-Phelps, K. Tracer diffusion in F-actin and Ficoll mixtures. Toward a model for cytoplasm. Biophys. J. 58, 31-43 (1990).

5. Moeendarbary, E. et al. The cytoplasm of living cells behaves as a poroelastic material. Nat. Mater. 12, 253-261 (2013).

6. Luby-Phelps, K. Cytoarchitecture and physical properties of cytoplasm: volume, viscosity, diffusion, intracellular surface area. Int. Rev. Cytol. 192, 189-221 (2000).

7. Wojcieszyn, J. W., Schlegel, R. A., Wu, E.-S. \& Jacobson, K. A. Diffusion of injected macromolecules within the cytoplasm of living cells. Proc. Natl Acad. Sci. USA 78, 4407-4410 (1981).

8. Tabaka, M., Kalwarczyk, T., Szymanski, J., Hou, S. \& Holyst, R. The effect of macromolecular crowding on mobility of biomolecules, association kinetics, and gene expression in living cells. Front. Phys. 2, 54 (2014).

9. Hofling, F. \& Franosch, T. Anomalous transport in the crowded world of biological cells. Rep. Prog. Phys. 76, 046602 (2013).

10. Luby-Phelps, K., Taylor, D. L. \& Lanni, F. Probing the structure of cytoplasm. J. Cell Biol. 102, 2015-2022 (1986).

11. Luby-Phelps, K., Castle, P. E., Taylor, D. L. \& Lanni, F. Hindered diffusion of inert tracer particles in the cytoplasm of mouse 3T3 cells. Proc. Natl Acad. Sci. USA 84, 49104913 (1987).

12. Jacobson, K. \& Wojcieszyn, J. The translational mobility of substances within the cytoplasmic matrix. Proc. Natl Acad. Sci. USA 81, 6747-6751 (1984).

13. Yokoe, H. \& Meyer, T. Spatial dynamics of GFP-tagged proteins investigated by local fluorescence enhancement. Nat. Biotechnol. s, 1252-1256 (1996).

14. Klann, M. T., Lapin, A. \& Reuss, M. Stochastic simulation of signal transduction: impact of the cellular architecture on diffusion. Biophys. J. 96, 5122-5129 (2009).

15. Banks, D. S. \& Fradin, C. Anomalous diffusion of proteins due to molecular crowding. Biophys. J. 89, 2960-2971 (2005).

16. Regner, B. M. et al. Anomalous diffusion of single particles in cytoplasm. Biophys. J. 104, 1652-1660 (2013).

17. Nelson, S. R., Ali, M. Y., Trybus, K. M. \& Warshaw, D. M. Random walk of processive, quantum dot-labeled myosin Va molecules within the actin cortex of COS-7 cells. Biophys. J. 97, 509-518 (2009).

18. Dix, J. A. \& Verkman, A. S. Crowding effects on diffusion in solutions and cells. Annu. Rev. Biophys. 37, 247-263 (2008).

19. Weiss, M., Elsner, M., Kartberg, F. \& Nilsson, T. Anomalous subdiffusion is a measure for cytoplasmic crowding in living cells. Biophys. J. 87, 3518-3524 (2004).

20. Keren, K., Yam, P. T., Kinkhabwala, A., Mogilner, A. \& Theriot, J. A. Intracellular fluid flow in rapidly moving cells. Nat. Cell Biol. 11, 1219-1224 (2009).

21. Barkai, E., Garini, Y. \& Metzler, R. Strange kinetics of single molecules in living cells. Phys. Today 65, 29-35 (2012).

22. Fulton, A. B. How crowded is the cytoplasm? Cell 30, 345-347 (1982).

23. Berry, H. \& Chaté, H. Anomalous diffusion due to hindering by mobile obstacles undergoing Brownian motion or Orstein-Ulhenbeck processes. Phys. Rev. E 89, 022708 (2014).

24. Guigas, G., Kalla, C. \& Weiss, M. The degree of macromolecular crowding in the cytoplasm and nucleoplasm of mammalian cells is conserved. FEBS Lett. 581, 50945098 (2007).

25. Pinaud, F., Clarke, S., Sittner, A. \& Dahan, M. Probing cellular events, one quantum dot at a time. Nat. Methods 7, 275-285 (2010). 
402

403

404

405

406

407

408

409

410

411

412

413

414

415

416

417

418

419

420

421

422

423

424

425

426

427

428

429

430

431

432

433

434

435

436

437

438

439

440

441

442

26. Clarke, S. et al. Covalent monofunctionalization of peptide-coated quantum dots for single-molecule assays. Nano Lett. 10, 2147-2154 (2010).

27. Okada, C. Y. \& Rechsteiner, M. Introduction of macromolecules into cultured mammalian cells by osmotic lysis of pinocytic vesicles. Cell 29, 33-41 (1982).

28. Nandi, A., Heinrich, D. \& Lindner, B. Distributions of diffusion measures from a local mean-square displacement analysis. Phys. Rev. E 86, 021926 (2012).

29. Luby-Phelps, K. et al. A novel fluorescence ratiometric method confirms the low solvent viscosity of the cytoplasm. Biophys. J. 65, 236-242 (1993).

30. Fushimi, K. \& Verkman, A. S. Low viscosity in the aqueous domain of cell cytoplasm measured by picosecond polarization microfluorimetry. J. Cell Biol. 112, 719-725 (1991).

31. Récamier, V. et al. Single cell correlation fractal dimension of chromatin. A framework to interpret 3D single molecule super-resolution. Nucleus 5, 75-84 (2014).

32. Metzler, R., Jeon, J.-H., Cherstvy, A. G. \& Barkai, E. Anomalous diffusion models and their properties: non-stationarity, non-ergodicity, and ageing at the centenary of single particle tracking. Phys. Chem. Chem. Phys. 16, 24128-24164 (2014).

33. Meroz, Y. \& Sokolov, I. M. A toolbox for determining subdiffusive mechanisms. Phys. Rep. 573, 1-29 (2015).

34. Weigel, A. V., Simon, B., Tamkun, M. M. \& Krapf, D. Ergodic and nonergodic processes coexist in the plasma membrane as observed by single-molecule tracking. Proc. Natl Acad. Sci. USA 108, 6438-6443 (2011).

35. Schulz, J. H. P., Barkai, E. \& Metzler, R. Aging effects and population splitting in singleparticle trajectory averages. Phys. Rev. Lett. 110, 020602 (2013).

36. Liße, D. et al. Monofunctional stealth nanoparticle for unbiased single molecule tracking inside living cells. Nano Lett. 14, 2189-2195 (2014).

37. Saxton, M. J. Anomalous diffusion due to obstacles: a Monte Carlo study. Biophys. J. 66, 394-401 (1994).

38. Saxton, M. J. Anomalous diffusion due to binding: a Monte Carlo study. Biophys. J. 70, 1250-1262 (1996).

39. Di Rienzo, C., Piazza, V., Gratton, E., Beltram, F. \& Cardarelli, F. Probing short-range protein Brownian motion in the cytoplasm of living cells. Nat. Commun. 5, 5891 (2014).

40. Del Pino, P. et al. Protein corona formation around nanoparticles - from the past to the future. Mater. Horiz. 1, 301-313 (2014).

41. Tabei, S. M. A. et al. Intracellular transport of insulin granules is a subordinated random walk. Proc. Natl Acad. Sci. USA 110, 4911-4916 (2013).

42. Janson, L. W., Ragsdale, K. \& Luby-Phelps, K. Mechanism and size cutoff for steric exclusion from actin-rich cytoplasmic domains. Biophys. J. 71, 1228-1234 (1996).

43. Guo, M. et al. Probing the stochastic, motor-driven properties of the cytoplasm using force spectrum microscopy. Cell 158, 822-832 (2014).

44. Tseng, Y., Kole, T. P. \& Wirtz, D. Micromechanical mapping of live cells by multipleparticle-tracking microrheology. Biophys. J. 83, 3162-3176 (2002).

45. $\mathrm{Hu}, \mathrm{J}$. et al. Size- and speed-dependent mechanical behavior in living mammalian cytoplasm. Proc. Natl Acad. Sci. USA 114, 9529-9534 (2017).

46. Delehanty, J. B., Mattoussi, H. \& Medintz, I. L. Delivering quantum dots into cells: strategies, progress and remaining issues. Anal. Bioanal. Chem. 393, 1091-1105 (2009).

47. Etoc, F. et al. Subcellular control of Rac-GTPase signalling by magnetogenetic manipulation inside living cells. Nat. Nanotechnol. 8, 193-198 (2013).

\section{Acknowledgment}


We acknowledge Maïté Coppey-Moisan (Institut Jacques Monod) for providing NIH 3T3 and hMSC cells, Bruno Goud (Institut Curie) for RPE-1 cells, and Sébastien Letard and Patrice Dubreuil (Centre de Recherche en Cancérologie de Marseille) for HDFa cells. E.B. acknowledges the Ecole Doctorale Frontières du Vivant (FdV) - Programme Bettencourt for financial support. MD and MC acknowledge financial support from French National Research Agency (ANR) Paris-Science-Lettres Program (ANR-10-IDEX-0001-02 PSL), Labex CelTisPhyBio (ANR-10-LBX-0038), the Human Frontier Science Program (grant no. RGP0005/2007) and the France-BioImaging infrastructure supported by ANR Grant ANR10-INSB-04 (Investments for the Future). This project has received funding from the European Union's Horizon 2020 Research and Innovation Programme under grant agreement No 686841 (MAGNEURON).

455

456

457

458

459

460

461

462

463

464

465

466

467

468

469

470

471

472

473

474

475

476

477

478

479

480

481

482

483

484

485

486

487

488

489

490

491

492

\section{Author contributions}

F.E., M.D., and MC conceived the study. F.E. performed experiments and numerical simulations. F.E. and D.N. did ferritin NPs experiments in HeLa cells. A.S. executed early experiments. C.V. conducted the NPs release validation experiments. E.B. performed RPE-1 and hMSC experiments. D.N. collected HDFa data. D.L. and J.P. provided reagents. F.E., E.B., and M.C. analysed the data. All authors discussed the results and commented on the manuscript. F.E., D.N., M.D., and M.C. wrote the manuscript.

\section{Competing financial interests}

The authors declare no competing financial interests.

\section{Methods}

Cell culture. HeLa and NIH $3 \mathrm{~T} 3$ cells were cultured in $1.0 \mathrm{~g} \cdot \mathrm{l}^{-1}$ glucose, phenol-red free DMEM (11054020, Gibco) supplemented with 10\% (v/v) FBS (10270106, Gibco), 1× GlutaMAXTM $^{\mathrm{TM}}$ (35050061, Gibco), and $100 \mathrm{U} \cdot \mathrm{ml}^{-1}$ of penicillin/streptomycin (15140122, Gibco). Primary normal, adult Human Dermal Fibroblast (HDFa, PCS-201-012 ${ }^{\mathrm{TM}}$, ATCC ${ }^{\circledR}$ ) were cultured in $4.5 \mathrm{~g} \cdot \mathrm{1}^{-1}$ glucose, phenol-red free DMEM (31053028, Gibco) supplemented with 10\% (v/v) FCS (CVFSVF00-01, Eurobio), 1 mM Sodium Pyruvate (11360070, Gibco), $100 \mathrm{U} \cdot \mathrm{ml}^{-1}$ penicillin/streptomycin $\left(15140122\right.$, Gibco), and 1× GlutaMAX ${ }^{\mathrm{TM}}$ (35050061, Gibco). Retinal Pigmented Epithelial (RPE-1) cells were cultivated using DME/F-12 medium (D8437, Sigma-Aldrich) supplemented with 10\% (v/v) FBS (10270106, Gibco). Cells were passed every 3 days when reaching 80-90\% confluency. Human Mesenchymal Stem Cells (hMSC, MSC-001F, StemCell) were cultivated with MesenCult ${ }^{\mathrm{TM}}$ proliferation kit (05411, StemCell) in flask coated with type I collagen (C8919, Sigma-Aldrich). Cells were passed every 10 days when reaching $70 \%$ confluency. All cells were maintained at $37^{\circ} \mathrm{C}$ in humidified, $\mathrm{CO}_{2}$-controlled $(5 \%)$ incubators. HeLa cells were purchased from ATCC. NIH 3T3 and hMSC cells were provided by Maïté Coppey-Moisan (Institut Jacques Monod) and originally obtained from ATCC and StemCell, respectively. RPE-1 cells were provided by Bruno Goud (Institut Curie) and originally obtained from ATCC. HDFa cells were provided by Sébastien Letard and Patrice Dubreuil (Centre de Recherche en Cancérologie de Marseille) and originally obtained from ATCC. 
Nanoparticles. The following quantum dots have been purchased from Thermo Fisher

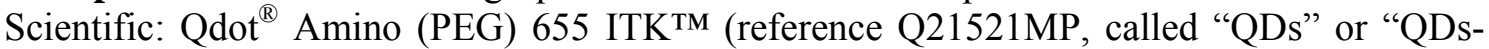
PEG-NH ${ }_{2}$ " in the text); Qdot ${ }^{\circledR} 655$ ITK ${ }^{\mathrm{TM}}$ Carboxyl (reference Q21321MP, "QDs-Itk-COOH" or "naked QDs" in the text); Qdot ${ }^{\circledR} 625$ Streptavidin Conjugate (reference Q22063, "QDsstreptavidin" in the text); Qdot ${ }^{\circledR} 605$ ITK $^{\text {TM }}$ Streptavidin Conjugate (reference Q10001MP, "QDs-605" in Supplementary Information). "QDs-peptide" have been previously obtained in the $\mathrm{lab}^{48}$ and "QDs-COOH-PEG" were obtained by PEGylation of "QDs-Itk-COOH". The hydrodynamic radius of all quantum dots is $20 \mathrm{~nm}$ (Thermo Fisher Scientific, personal communication). Red-emitting polystyrene/polymethacrylate NPs of different diameters ("Rho-NPs" in the text) were made on our demand by micromod Partikeltechnologie GmbH (Germany): $15 \mathrm{~nm}$ plain $\mathrm{NH}_{2}$ (reference 30-01-151), $25 \mathrm{~nm}$ streptavidin (reference 30-19251), $50 \mathrm{~nm}$ streptavidin (reference 30-19-501), $75 \mathrm{~nm}$ streptavidin (reference 30-19-751). Characterization of Rho-NPs size is reported in Supplementary Fig. 10. Ferritin-PEGAtto647N NPs ("ferritin NPs" in the text) have been previously obtained in the lab ${ }^{36}$. The zeta potential of NPs is reported in Supplementary Table 3.

509

510

511

512

513

514

515

516

517

518

519

520

521

522

523

524

525

526

527

528

529

530

531

532

533

534

535

536

537

538

Nanoparticles internalization. NPs internalization via pinocytosis in HeLa, NIH 3T3, and HDFa cells was done on adherent cells, plated on glass coverslips the day before experiments, following the manufacturer's procedure (Influx ${ }^{\mathrm{TM}}$ reagent, I14402 Thermo Fisher Scientific). Cells were incubated 10 minutes at $37^{\circ} \mathrm{C}$ with hypertonic medium containing the desired NPs at a concentration of $\sim 15 \mathrm{nM}$. Then, coverslips were incubated vertically for two minutes in hypotonic medium (serum free medium and deionized water in a 6:4 ratio) and washed extensively with fresh medium. Pinocytosis in RPE-1 and hMSC cells was done using cells in suspension according to the advices of the Influx ${ }^{\mathrm{TM}}$ reagent provider. After being detached from culture dishes, cells were pelleted at $300 \times \mathrm{g}(\mathrm{RPE}-1)$ or $450 \times \mathrm{g}(\mathrm{hMSC})$ and then resuspended and incubated for 10 minutes with $20 \mu \mathrm{l}$ of hypertonic solution with NP concentrations on the order of 4 to $10 \mathrm{nM}$ for QDs-Itk-COOH, QD-PEG-NH , and ferritin NPs and of $90 \mathrm{nM}$ for $25 \mathrm{~nm}$ Rho-NPs. Next, $1 \mathrm{ml}$ of hypotonic medium was added and, 1 minute after addition, cells were pelleted for $60 \mathrm{~s}$. Total exposure to hypotonic medium ranged between $150 \mathrm{~s}$ and $180 \mathrm{~s}$. Cells were then re-suspended in fresh medium and washed at least three times. Finally, cells were plated on glass coverslip previously incubated (30 min at $37^{\circ} \mathrm{C}$ ) with $100 \mu \mathrm{g} \cdot \mathrm{ml}^{-1}$ type I collagen in PBS. In all cases, cells were let recover for at least one hour in the incubator before imaging. For microinjection experiments (Supplementary Fig. 7 and used for $75 \mathrm{~nm}$ Rho-NPs internalization), glass pipettes with $\sim 1 \mu \mathrm{m}$ diameter aperture were realized with a P-97 pipette puller (Sutter Instrument). Cell were injected using a semi-automatic microinjection system (Injectman II, Epperndorf) with $\sim 0.1 \mathrm{pl}$ of NPs solution ( $\sim 15 \mathrm{nM}$ in PBS), which represents about one tenth of the cell volume. Cell injection was done within a small coverslip area marked by drawing a circle below the coverslip. After injection, cells were placed in the incubator for one hour before imaging to ensure their recover and osmolarity re-equilibration. For experiments involving drug treatments, after QDs internalization, cells were incubated for one hour with the indicated concentration of drug freshly diluted from concentrated DMSO stocks.

Characterization of the NPs internalization. The osmotic shock applied during pinocytosis led to bursting of most of the $\sim 2 \mu \mathrm{m}$-diameter pinocytotic vesicles (about 8 vesicles were formed per cell) and to the delivery of 20 to 50 individual NPs per cell (Supplementary Fig. 1). After pinocytic treatment $\sim 70 \%$ of cells were viable and we did not observe any additional

541 toxicity due to NPs (Supplementary Fig. 2). Remaining unburst vesicles (at most 3 per cell,

542 Supplementary Fig. 1) were systematically excluded when selecting regions of interest for 
543

544

545

546

547

548

549

550

551

552

553

554

555

556

557

558

559

560

561

562

563

564

565

566

567

568

569

570

571

572

573

574

575

576

577

578

579

580

581

582

583

584

585

586

587

588

589

590

591

592

analysis (see Supplementary Video 1 and 2). Contrarily to NPs enclosed in unburst vesicles, NPs released in the cytosol were able to diffuse in the whole cytoplasm, without entering into the nucleus, and to efficiently target specific cellular components (Supplementary Fig. 3). In the case of QDs, we observed blinking of the fluorescence signal, a typical signature of individual emitters (Supplementary Fig. 4 and Supplementary Video 3) indicative of monodispersed particles. When NPs of two distinct colours were co-internalized, no co-localization or correlated dynamics were observed (Supplementary Fig. 5 and Supplementary Videos 4 and 5). We did not find significant differences in the behaviour of both $25 \mathrm{~nm}$ Rho-NPs and QDs when performing experiments in two different mammalian cell lines (HeLa and 3T3, Supplementary Fig. 6) or when NPs were microinjected in cells, as an alternative internalization method (Supplementary Fig. 7).

Imaging. Cells were imaged using thermalized $\left(37^{\circ} \mathrm{C}\right)$, atmosphere-controlled $\left(5 \% \mathrm{CO}_{2}\right)$ inverted microscopes, using high N.A. $100 \times$ oil-immersion objectives, and back-illuminated EMCCDs for image acquisition. After NPs internalization, we selected 20 to 30 regions of interest $(\sim 150 \times 150$ pixels $)$ away from the cell nucleus and that did not contain unburst vesicles, which were easily distinguished from single, diffusing NPs (see Supplementary Video 1 and 2) thanks to their brightness and size (Supplementary Fig. 1). Each recording lasted 1,000 frames and at least 12 cells were imaged for each experimental condition from at least three different sample preparations. In HeLa, NIH 3T3, RPE-1, and hMSC cells experiments, time-lapse images of diffusing QDs were recorded using a Xe lamp for UV epifluorescence illumination, a bandpass filter (FF02-641/75-25, Semrock), and an exposure time of $30 \mathrm{~ms}$. Rho-NPs were imaged with an exposure time of $10 \mathrm{~ms}$ under wide field laser excitation $(561 \mathrm{~nm})$ using a long pass dichroic filter with cut off at $573 \mathrm{~nm}$ (FF573-Di0125x36, Semrock) and an emission filter centred at $593 \mathrm{~nm}$ (FF01-593/46-25, Semrock). Recordings of rapidly diffusing ferritin NPs were performed with $2 \mathrm{~ms}$ integration time using a previously described setup ${ }^{49}$ employing a $640 \mathrm{~nm}$ laser and a long pass emission filter (BLP01-635R-25, Semrock). Two colours experiments (Supplementary Fig. 5 and Supplementary Video 4 and 5) were executed using a dual-view beam splitter (Photometrics). HDFa experiments have been conducted using HILO illumination ${ }^{50}$ and a multi-band dichroic for laser excitation (Di01-R405/488/561/635-25x36, Semrock). QDs have been excited with a $405 \mathrm{~nm}$ laser and imaged through a bandpass filter (FF01-676/37-25, Semrock) using $30 \mathrm{~ms}$ exposure time. Rho-NPs have been observed under $561 \mathrm{~nm}$ laser excitation using a bandpass filter (616/57, 489074, Zeiss) and $10 \mathrm{~ms}$ exposure time, while ferritin NPs have been imaged with a $642 \mathrm{~nm}$ laser using a bandpass filter (FF01-676/37-25, Semrock) and $5 \mathrm{~ms}$ exposure time.

Trajectory analysis. Two-dimensional projection (on the $x-y$ plane) of cytoplasmic diffusion of single NPs was recorded and analysed. Motion along the optical axis (z) was not considered under the assumption that all directions are equivalent for diffusion within an isotropic medium. Trajectories were reconstructed with the MATLAB (MathWorks) routine MTT, which accounts for blinking in the trajectories ${ }^{51}$. Each dataset contained at least 140 trajectories lasting more than 40 time points. Individual trajectories were analysed with home written MATLAB routines. Two-dimensional time-averaged mean squared displacements were calculated using the formula $\left\langle r^{2}(\tau)\right\rangle_{\mathrm{T}}=\left\langle[x(t+\tau)-x(t)]^{2}+[y(t+\tau)-y(t)]^{2}\right\rangle_{\mathrm{T}}$, where the average is done over all points of the trajectory separated by a time interval $\tau$ (sliding average). To compute the time-averaged mean square displacement $\left\langle r_{60 \mathrm{~ms}}^{2}\right\rangle_{\mathrm{T}}$ over 60 $\mathrm{ms}$, we first performed a linear fit on the first points of the time-averaged MSD curves to remove the intercept at time 0 , which is on the order of $60 \mathrm{~nm}$ for QDs (representative dataset for NPs intensities). As described by Michalet ${ }^{52}$, the accuracy in the determination of the 
slope of the MSD, which equals $4 D$ in the present case, is related to the number of points used for the MSD fit. We compared the results obtained considering different numbers of points for the fit (from 2 to 10) and found no significant variation in the $\alpha-D$ maps (Supplementary Fig. 8a). Thus, we chose to fit the taMSDs curves up to $60 \mathrm{~ms}$ ( 2 to 6 points depending on the data set), in order to make the comparison between all NPs relevant. The obtained instantaneous diffusivities were called $D_{60 \mathrm{~ms}}$. The ensemble average of taMSDs was calculated following the same routine as in Ref. 34. The exponent $\alpha$ was obtained as single fitting parameter from the log-log plot of taMSD using a general diffusion model $\left\langle r^{2}\right\rangle_{\mathrm{T}} \sim t^{\alpha}$. We acknowledge that the offset of the MSD, due to the static and dynamic errors, should be removed when fitting $\alpha$ either by performing a fit with one or two additional free parameters ${ }^{53}$ or by removing a constant value. However, we found that the low statistics in the number of frame for individual trajectories prevented us from performing a unconstrained multiparameter fit. Doing so, we obtained unrealistic values for $\alpha$ and the errors. Yet, for all

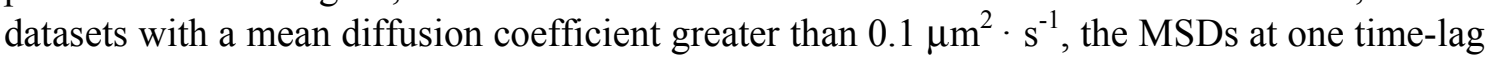
are at least one order magnitude larger than the pointing error and, thus, the bias in our estimation can be neglected. $\alpha-D_{60 \mathrm{~ms}}$ maps were generated using the MATLAB function scattercloud, which smoothens two-dimensional histograms. The contour lines (percentiles of the probability density) were obtained using the MATLAB routine ksbivardens, which performs a bivariate kernel density estimation, and the function quantile. For analysis of RhoNP subpopulations, we used a $k$-mean algorithm to cluster data points in the $\alpha-D_{60 \mathrm{~ms}}$ plane. We used four clusters and grouped together the two clusters with largest $D_{60 \mathrm{~ms}}$ values to evaluate the fast population and the two with the smallest $D_{60 \mathrm{~ms}}$ values for the slow population (Supplementary Fig. 9).

Trajectory simulations. Brownian trajectories were simulated using MATLAB routines. The time lag was fixed at $\Delta t=10 \mathrm{~ms}$. Elementary $\Delta x, \Delta y$, and $\Delta z$ steps were drawn from a normal distribution with zero mean and variance equal to $\sqrt{2 \cdot D \cdot \Delta t}$, the diffusion coefficient $D$ was chosen to be $10 \mu \mathrm{m}^{2} \cdot \mathrm{s}^{-1}$ (and $0.25 \mu \mathrm{m}^{2} \cdot \mathrm{s}^{-1}$ for Supplementary Fig. 13c). Initially, one particle was randomly distributed in a representative box of 10 by $10 \mu \mathrm{m}$ and $1 \mu \mathrm{m}$ height to fit the experimental volume of detection, including the limited focal depth of the microscope. The particle was then allowed to diffuse. Boundaries were considered to be reflective to take into account the exchange of particles in the observation field at equilibrium. However, each time a particle reached the boundaries, the trajectory was interrupted and a new one started to mimic particle loss by the tracking software; otherwise, trajectories were interrupted when they reached 500 time steps. Overall, the number of trajectories (4000) as well as the trajectory length distribution matched the experimental data. Simulated trajectories were subsequently analysed using the same routines used for the experimental ones. For CTRW trajectories, the boundary conditions were relaxed in order to keep ideal, perfectly inert particles, with a diffusion coefficient of $10 \mu \mathrm{m}^{2} \cdot \mathrm{s}^{-1}$, in the computational volume for a time sufficient to generate long enough trajectories. Specifically, the box linear dimensions were expanded by a factor 10 . Between subsequent steps, corresponding to elementary jumps lasting $10 \mathrm{~ms}$ with $D=10 \mu \mathrm{m}^{2} \cdot \mathrm{s}^{-1}$, the particle was immobilized for a random waiting time $T$ sampled form the cumulative distribution $F(T>t)=\left(t_{0} / t\right)^{\gamma}$.

Code availability. Custom written MATLAB routines (for MATLAB version R2015a) for trajectory analysis, numerical simulation, and density plot generation will be made available to readers by the authors upon reasonable request. 
641

642

643

644

645

646

647

648

649

650

651

652

653

654

655

656

657

658

659

660

661

662

663

664

665

666

667

668

669

670

671

672

673

674

675

676

677

678

679

680

681

682

683

684

685

686

687

688

689

690

Data availability. All the data supporting the findings of this study, within the article and its supplementary information, will be made available to readers by the authors upon reasonable request.

\section{References}

48. Clarke, S., Tamang, S., Reiss, P. \& Dahan, M. A simple and general route for monofunctionalization of fluorescent and magnetic nanoparticles using peptides. Nanotechnology 22, 175103 (2011).

49. Normanno, D. et al. Probing the target search of DNA-binding proteins in mammalian cells using TetR as model searcher. Nat. Commun. 6, 7357 (2015).

50. Tokunaga, M., Imamoto, N. \& Sakata-Sogawa, K. Highly inclined thin illumination enables clear single-molecule imaging in cells. Nat. Methods 5, 159-161 (2008).

51. Sergé, A., Bertaux, N., Rigneault, H. \& Marguet, D. Dynamic multiple-target tracing to probe spatiotemporal cartography of cell membranes. Nat. Methods 5, 687-694 (2008).

52. Michalet, X. Mean square displacement analysis of single-particle trajectories with localization error: Brownian motion in an isotropic medium. Phys. Rev. E 82, 041914 (2010).

53. Backlund, M. P., Joyner, R. \& Moerner, W. E. Chromosomal locus tracking with proper accounting of static and dynamic errors. Phys. Rev. E 91, 062716 (2015).

\section{Figure Captions}

Figure 1. NPs of similar size have different diffusive signatures.

Two types of NPs (25nm Rho-NPs and QDs) were chosen as an example to show that tracers of similar size $(\sim 25 \mathrm{~nm})$ can have different cytoplasmic diffusion properties. a, Distribution of the instantaneous diffusivity coefficient $D_{60 \mathrm{~ms}}$ for $25 \mathrm{~nm}$ Rho-NPs in HeLa cells $(N=1314$ trajectories). b, Density plot in the $\alpha-D_{60 \mathrm{~ms}}$ plane for $25 \mathrm{~nm}$ Rho-NPs. Colour code represents the local density of trajectories normalized between 0 (blue) and 1 (red). Black dots correspond to individual trajectories $(N=309$ trajectories lasting more than 40 frames, out of 1314 recorded trajectories). Black lines (contours) indicate 25, 50, and 75 percentiles. White, dashed lines are a guide to the eye. c, Distribution of $D_{60 \mathrm{~ms}}$ for QDs in HeLa cells $(N=1696$ trajectories). d, Density plot in the $\alpha-D_{60 \mathrm{~ms}}$ plane for QDs ( $N=525$ out of 1696 trajectories). e, Cumulative distributions of $D_{60 \mathrm{~ms}}$ for QDs in glycerol (black, $N=5118$ trajectories), in HeLa cells (green, $N=1696$ trajectories), and for $25 \mathrm{~nm}$ Rho-NPs in HeLa cells (blue, $N=$ 1314 trajectories). f, Density plot in the $\alpha-D_{60 \mathrm{~ms}}$ plane for QDs in $80 \%(\mathrm{v} / \mathrm{v})$ glycerol $(N=273$ out of 5118 trajectories). $D_{60 \mathrm{~ms}}$ is expressed in $\mu \mathrm{m}^{2} \cdot \mathrm{s}^{-1}$.

Figure 2. Cytoplasmic mobility is strongly reduced for NPs above $50 \mathrm{~nm}$ in size.

Representation of Rho-NPs trajectories in the $\alpha-D_{60 \mathrm{~ms}}$ plane in HeLa cells for a, $15 \mathrm{~nm}$ RhoNPs $(N=186), \mathbf{b}, 25 \mathrm{~nm}$ Rho-NPs $(N=309)$, c, $50 \mathrm{~nm}$ Rho-NPs $(N=837)$, and d, $75 \mathrm{~nm}$ RhoNPs $(N=202)$. $D_{60 \mathrm{~ms}}$ is expressed in $\mu \mathrm{m}^{2} \cdot \mathrm{s}^{-1}$.

Figure 3. QDs show intrinsic sub-diffusive behaviour and ergodicity breaking.

a, Ensemble average of time-averaged MSDs, $\left\langle\left\langle r^{2}\right\rangle_{\mathrm{T}}\right\rangle_{\mathrm{E}}$, in log-log scale obtained considering all individual time-averaged MSDs ( $N=1696$ trajectories). The sub-diffusive behaviour of QDs is evidenced by the deviation of QDs MSD curve (red data points) from the blue straight line corresponding to Brownian diffusion (slope $=1$ ). Data points represent mean values and error bars are standard error of the mean. $\mathbf{b},\left\langle\left\langle r^{2}\right\rangle_{\mathrm{T} \geq \mathrm{N} \Delta \mathrm{t}}\right\rangle_{\mathrm{E}}$ computed by considering only 
trajectories lasting more than 30 (blue crosses, $N=690$ trajectories), 70 (green circles, $N=$ 692289 trajectories), or 150 (red triangles, $N=100$ trajectories) frames. MSD curves of QDs 693 trajectories homogeneous in length are linear in log-log scale, with an identical slope $\sim 0.82$. 694 c-d, Temporal (top) versus ensemble (bottom) distributions of $D_{60 \mathrm{~ms}}$ for QDs in (c) HeLa cells $695(N=650$ trajectories lasting more than 40 frames, out of 1696 trajectories $)$ and (d) in $80 \%$ (v/v) glycerol ( $N=1512$ trajectories lasting more than 10 frames, out of 5118 trajectories). Temporal distribution in (c) proceeds from Fig. 1c, while the ensemble distribution was obtained through the following procedure: one value of the ensemble-averaged MSDs was built by averaging the 60 ms-square displacements for all NPs detected at a given time point of the acquisition, and the distribution of ensemble-averaged MSDs was obtained by performing this average for all time points of the acquisition. e, Cumulative distribution of $\log \left(D_{60 \mathrm{~ms}}\right)$ for QDs with five different surface coatings in HeLa cells: QDs-Itk-COOH (black, $N=915$ trajectories), QDs-peptide (red, $N=785$ trajectories), QDs-COOH-PEG (green, $N=$ 6531 trajectories), QDs-streptavidin (blue, $N=685$ trajectories), QDs-PEG- $\mathrm{NH}_{2}$ (purple, $N=$ 1696 trajectories). Note that QDs-PEG-NH $\mathrm{NH}_{2}$ are referred as QDs in the text. $D_{60 \mathrm{~ms}}$ is expressed in $\mu \mathrm{m}^{2} \cdot \mathrm{s}^{-1}$.

\section{Figure 4. A minimal CTRW model recapitulates all the different NPs diffusive behaviours observed.}

Representation in the $\alpha-D_{60 \mathrm{~ms}}$ plane of simulated trajectories obtained using a simple CTRW model with different values of $\gamma: \infty, 10,2,1,0.5$, and 0.1 . For large values of $\gamma$, corresponding to perfectly inert probes, diffusion is fast $\left(D_{60 \mathrm{~ms}} \sim 10 \mu \mathrm{m}^{2} \cdot \mathrm{s}^{-1}\right)$ and Brownian. When $\gamma$ is equal to 1 (the critical value of the immobilisation time to be integrable), diffusion is slowed down by one order of magnitude $\left(D_{60 \mathrm{~ms}} \sim 1 \mu \mathrm{m}^{2} \cdot \mathrm{s}^{-1}\right)$ but still Brownian, as observed for RhoNPs. When $\gamma$ is further decreased $(\gamma=0.5)$, the immobilisation time diverges, the diffusion coefficient drops by one more order of magnitude and sub-diffusive behaviour, together with the "coma" shape in the $\alpha-D_{60 \mathrm{~ms}}$ plane, emerges, as observed in the case of QDs-PEG-NH $\left(D_{60 \mathrm{~ms}} \sim 0.1 \mu \mathrm{m}^{2} \cdot \mathrm{s}^{-1}\right.$ and $\left.\alpha \sim 0.8\right)$. The case $\gamma=0.1$ corresponds to strongly sub-diffusing objects immobilised almost all the time, as "naked" QDs (QDs-Itk-COOH). In each density map, $N=4000$ simulated trajectories. $D_{60 \mathrm{~ms}}$ is expressed in $\mu \mathrm{m}^{2} \cdot \mathrm{s}^{-1}$.

Figure 5. NPs diffusivity can be ranked by the strength of non-specific interactions.

Representation in the $\alpha-D_{60 \mathrm{~ms}}$ plane of trajectories obtained from experiments with different NPs of similar size $(\sim 25 \mathrm{~nm})$ in HeLa cells, in adult Human Dermal Fibroblasts (HDFa), in Retinal Pigmented Epithelial (RPE-1) cells, and in human Mesenchymal Stem Cells (hMSC). Data are sorted as a function of the instantaneous diffusivity coefficient $D_{60 \mathrm{~ms}}$. The minimal CTRW model suggests that such an ordering correlates with the strength of NPs non-specific interactions with immobile, or slowly moving, intracellular components. The number $N$ of trajectories included the $\alpha-D_{60 \mathrm{~ms}}$ maps is (from top to bottom): 687, 309, 525, 389 for HeLa; 831, 1622, 237, 113 for HDFa; 469, 747, 466, 142 for RPE-1; and 645, 266, 168, 296 for hMSC. $D_{60 \mathrm{~ms}}$ is expressed in $\mu \mathrm{m}^{2} \cdot \mathrm{s}^{-1}$.

\section{Figure 6. General model of cytosolic mobility.}

Objects (red spheres) that have size larger than the cytoskeleton mesh size (between 50 and $70 \mathrm{~nm}$ ) are trapped in a viscoelastic medium and experience restricted diffusion. On time scales of seconds, such large objects are confined, whereas they will move at longer time scales thanks to the remodelling of internal structures (cytoskeleton, endomembranes). Objects with size smaller than the pore size diffuse in a liquid phase whose apparent viscosity depends on the strength of the biochemical interactions. If objects are perfectly inert, they 
740 diffuse freely without hindering due to obstacles. The apparent viscosity is then slightly 741 higher than that of water. When objects start to interact with immobile and slowly moving 742 intracellular components, diffusion is slowed down but remains Brownian if the 743 immobilisation time does not exceed the time scale over which diffusion is measured. If the 744 strength of interactions increases up to the point that the immobilisation time can be longer 745 than the observation time, the diffusion is further slowed down and become anomalous. 


\section{Non-specific interactions govern cytosolic diffusion of nano-sized objects in mammalian cells}

Fred Etoc $^{1, \S}$, Elie Balloul ${ }^{1, \dagger}$, Chiara Vicario ${ }^{1, \dagger, \S}$, Davide Normanno ${ }^{1,2, *}$, Domenik Liße ${ }^{3}$, Assa Sittner ${ }^{4}$, Jacob Piehler ${ }^{3}$, Maxime Dahan ${ }^{1, *}$, Mathieu Coppey ${ }^{1, *}$

${ }^{1}$ Laboratoire Physico-Chimie, Institut Curie, CNRS UMR168, PSL Research University, Université Pierre et Marie Curie-Paris 6. 11 rue Pierre et Marie Curie, 75248 Paris - Cedex 05, France

${ }^{2}$ Centre de Recherche en Cancérologie de Marseille, CNRS UMR7258, Inserm U1068, Aix-Marseille Université UM105, Institut Paoli-Calmettes. 27 boulevard Leï Roure, 13273 Marseilles - Cedex 09, France

${ }^{3}$ Division of Biophysics, Department of Biology, Osnabrück University. Barbarastraße 11, 49076 Osnabrück, Germany

${ }^{4}$ Department of Infectious Diseases, Israel Institute for Biological Research. POB 19, 7410001 Ness Ziona, Israel

$\dagger$ Equally contributed to this work

$\S$ current address:

Center for Studies in Physics and Biology, The Rockefeller University. 1230 York Avenue, New York, NY 10065, USA (F.E.). Centre for Genomic Regulation, The Barcelona Institute of Science and Technology. Carrer del Dr. Aiguader 88, 08003 Barcelona, Spain (C.V.)

* Correspondence should be addressed to D.N. (davide.normanno@inserm.fr), M.D. (maxime.dahan@curie.fr), and M.C. (mathieu.coppey@curie.fr) 


\title{
Supplementary Video Legends
}

\begin{abstract}
Supplementary Video 1
Representative video of $25 \mathrm{~nm}$ Rho-NPs diffusing in a HeLa cell after pinocytic loading. Three unburst vesicles can be seen at the top of the images: two very, close together, on the left and one, very bright, in the middle. These unburst vesicles are much larger than individual NPs and are basically immobile. Images were acquired every $10 \mathrm{~ms}$, the video is encoded at 30 frames per second (fps). Scale bar represents $10 \mu \mathrm{m}$.
\end{abstract}

\section{Supplementary Video 2}

Representative videos of $25 \mathrm{~nm}$ Rho-NPs diffusing in HeLa cells after pinocytic loading. Top row: crops of the field of view showing unburst vesicles. NPs encapsulated within these intact vesicles move very fast and cannot be tracked individually. Bottom row: examples of typical regions of interest chosen for NPs single-particle tracking analysis and selected in a way such that unburst vesicles were systematically excluded. The marked difference in the behaviour of free versus encapsulated NPs ensures a reliable discrimination between the two cases. Images were acquired every $10 \mathrm{~ms}$, the video is encoded at $30 \mathrm{fps}$. Scale bars represent $1 \mu \mathrm{m}$.

\section{Supplementary Video 3}

Representative video of QDs (QDs-PEG-NH $\mathrm{N}_{2}$ ) diffusing in a HeLa cell after pinocytic loading. QDs clearly show on-off fluctuations (blinking) of the fluorescence signal (use Supplementary Fig. 4 as reference to more easily spot blinking particles). The blinking behaviour is a typical signature of individual emitters and indicates that QDs are mono-dispersed, confirming their escape from pinocytic vesicles and dismissing potential aggregations. Images were acquired every $30 \mathrm{~ms}$, the video is encoded at $15 \mathrm{fps}$. The counter indicates the frame number. Scale bar represents $2 \mu \mathrm{m}$.

\section{Supplementary Video 4}

Representative video of the simultaneous diffusion of two different types of QDs, QDs-605 emitting at $605 \mathrm{~nm}$ (green) and QDs-PEG-NH 2 emitting at $655 \mathrm{~nm}$ (red), after concomitant pinocytic internalization. The images clearly illustrate that in the two HeLa cells visible in the video there is no co-localization of the two probes, on the contrary of what expected if NPs would have been still encapsulated inside unburst vesicles. Images were acquired every $30 \mathrm{~ms}$, the video is encoded at $15 \mathrm{fps}$. Scale bar represents $5 \mu \mathrm{m}$.

\section{Supplementary Video 5}

The video shows the concurrent detection of the two types of QDs displayed in Supplementary Video 4: QDs-605 (red circles) and QDs-PEG-NH 2 (yellow circles). The images put well in evidence the absence of correlated dynamics in the mobility of the two probes, on the contrary of what expected if NPs would have been still encapsulated inside unburst vesicles. Images were acquired every $30 \mathrm{~ms}$, the video is encoded at $10 \mathrm{fps}$. Scale bar represents $5 \mu \mathrm{m}$. 


\section{Supplementary Figure 1}

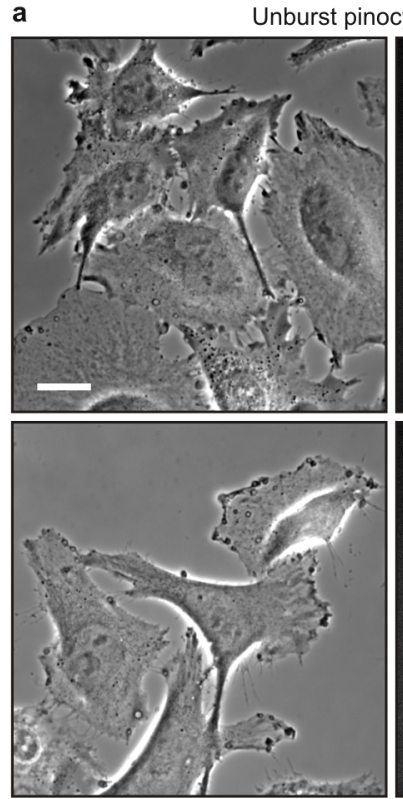

b

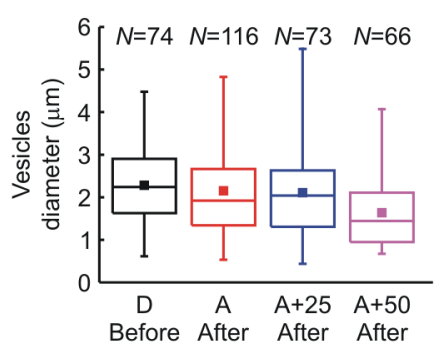

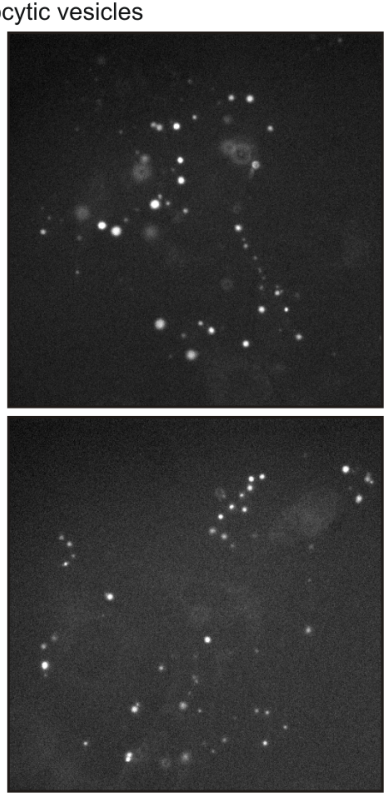

C

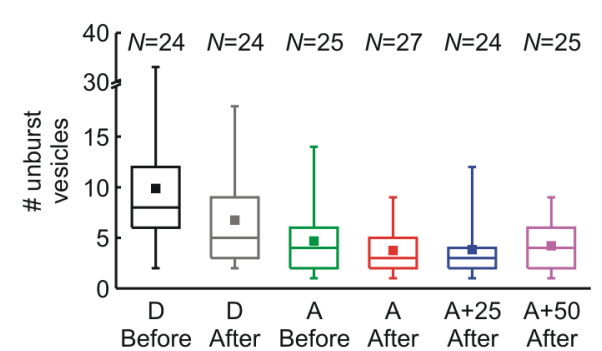

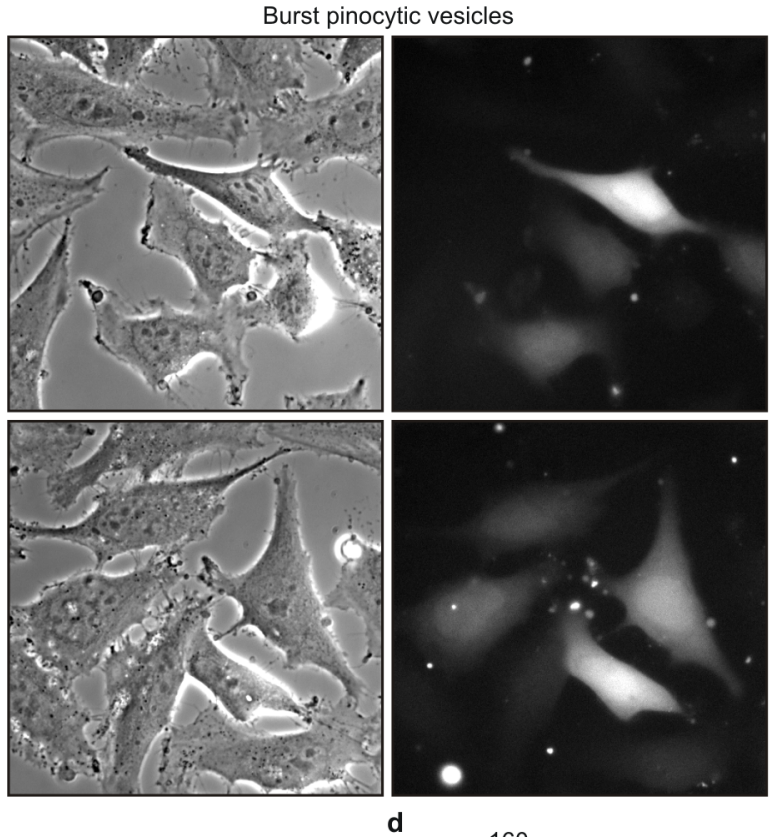

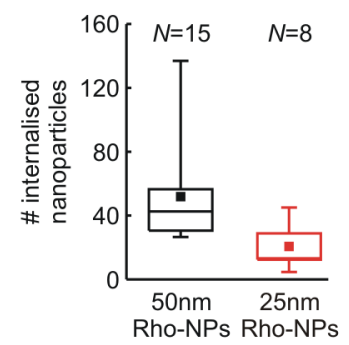

\section{Supplementary Figure 1 | Characterization of pinocytic internalization}

a) Formation and bursting of pinocytic vesicles. Left. Representative phase contrast and fluorescence images of HeLa cells containing unburst vesicles after pinocytic loading using FITC-Dextran (MW $150 \mathrm{kDa}$, concentration $\sim 5 \mathrm{nM}$ ). Several pinocytic vesicles are formed in each cell: they appear as bright spots in the fluorescence images and are also visible in phase contrast. The choice to use high molecular weight Dextran was instrumental and dictated by the fact that it prevented bursting of the vesicles permitting, therefore, to quantify by fluorescence imaging the size and number of vesicles formed during pinocytosis (see Panels b and c). In fact, because of their extreme sensitivity to osmolarity, pinocytic vesicles burst even upon a gentle wash or dilution of the hypertonic medium, which prevents to reduce the extracellular fluorescence background and, thus, to image intact vesicles when filled with NPs. Right. Representative phase contrast and fluorescence images of HeLa cells after pinocytic loading of Atto488 (MW $\sim 1 \mathrm{kDa}$, concentration $\sim 70 \mu \mathrm{M}$ ) used to quantify the number of vesicles remaining intact after osmotic shock (see Panel c). Of note, the presence in the field of view of unstained cells confirms that Atto488 was internalized via pinocytosis and not via passive diffusion. Each experiment has been repeated twice. Scale bar represents $20 \mu \mathrm{m}$. 
b) Quantification of pinocytic vesicles size. Box plots of pinocytic vesicles size quantified from fluorescence images after intensity thresholding. The size of intact vesicles was measured in the case of two different loading procedures: $10 \mathrm{~min}$ of Influx treatment in the presence of FITC-Dextran (black, "D Before"), and 10 min of Influx treatment in the presence of Atto488 (red, "A After") plus $25 \mathrm{~nm}$ (blue, "A+25 After") or $50 \mathrm{~nm}$ (magenta, "A+50 After") Rho-NPs followed by 2 min of hypotonic medium incubation. In all cases, vesicles ranged between about 1 and $4 \mu \mathrm{m}$ (with a mean size of $\sim 2 \mu \mathrm{m}$ ), thus, well above the point-spread-function of our imaging systems $(\sim 300 \mathrm{~nm})$, which ensures that they can be easily distinguished from diffraction-limited NPs. Boxes indicate 25 to $75 \%$ of the underlying distribution, central lines represent median values, solid squares denote mean values, vertical bars cover the interval from 1 to $99 \%$ of the distribution. $N$ represents the number of vesicles analysed.

c) Quantification of unburst vesicles number. Box plots of the number of intact vesicles per cell in the case of two different loading procedures: 10 min of Influx treatment in the presence of FITC-Dextran (black, "D Before", showing an average of 8 vesicles formed per cell) or of Atto488 (green, "A Before"), and 10 min of Influx treatment in the presence of FITC-Dextran (grey, "D After"), Atto488 (red, "A After") plus $25 \mathrm{~nm}$ (blue, "A+25 After") or $50 \mathrm{~nm}$ (magenta, "A+50 After") Rho-NPs followed by $2 \mathrm{~min}$ of hypotonic medium incubation (showing on average of at most 3 unburst vesicles per cell). Boxes indicate 25 to $75 \%$ of the underlying distribution, central lines represent median values, solid squares denote mean values, vertical bars cover the interval from 1 to $99 \%$ of the distribution. $N$ represents the number of cells analysed.

d) Quantification of the number of released NPs per cell. Box plots of the number of internalized nanoparticles into individual HeLa cells after $10 \mathrm{~min}$ of Influx treatment followed by 2 min of hypotonic medium incubation. We found $52 \pm 32$ NPs per cell (mean \pm SEM) in the case of 50nm Rho-NPs (black) and $21 \pm 13$ for $25 \mathrm{~nm}$ Rho-NPs (red). Boxes indicate 25 to $75 \%$ of the underlying distribution, central lines represent median values, solid squares denote mean values, vertical bars cover the interval from 1 to $99 \%$ of the distribution. $N$ represents the number of cells analysed. 


\section{Supplementary Figure 2}

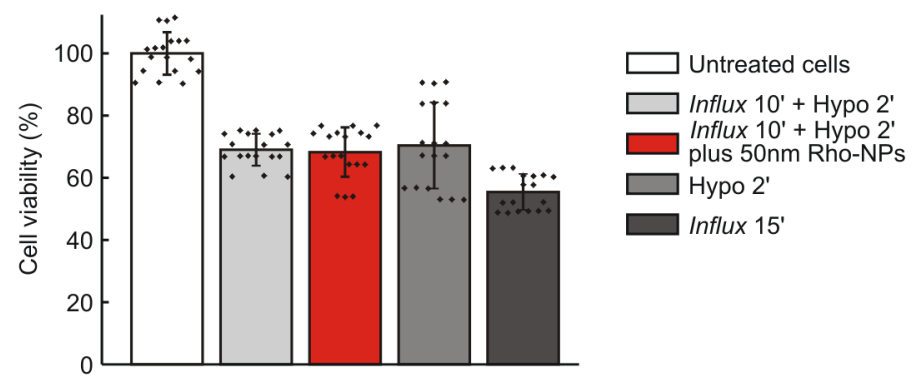

Supplementary Figure 2 | Cells are largely viable after pinocytic internalization of 50nm Rho-NPs

Cell viability was measured using a MTT-based kit (M5655, Sigma-Aldrich) following the manufacturer procedure. HeLa cells were plated on 96-well plates and were either left untreated (white) or treated with the standard pinocytosis procedure: 10 min Influx reagent followed by 2 min of hypotonic medium incubation. In the latter case, about $70 \%$ of cells survived to the protocol irrespectively of the absence (light grey) or the presence (red) of 50nm Rho-NPs, indicating no additional cellular toxicity of NPs. Incubation with only hypotonic medium for 2 min (Hypo) resulted in similar levels of cell viability (grey) while longer incubation with the Influx reagent (e.g., $15 \mathrm{~min}$ ) reduced cell viability to less than $60 \%$ (dark grey). Column values represent the average cell viability resulting from 18 independent wells (black diamonds) pulled together from three different experimental replicas. Error bars are standard deviations. 


\section{Supplementary Figure 3}
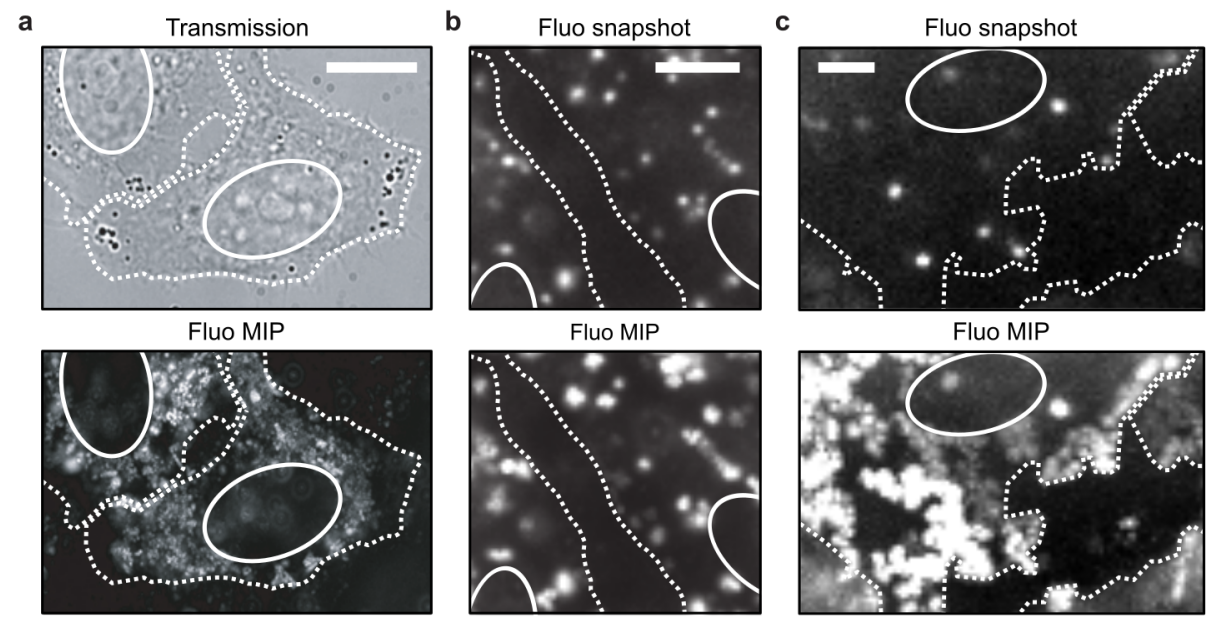

d

$\mathrm{DIC}$

mCherry channel

GFP channel

Merge
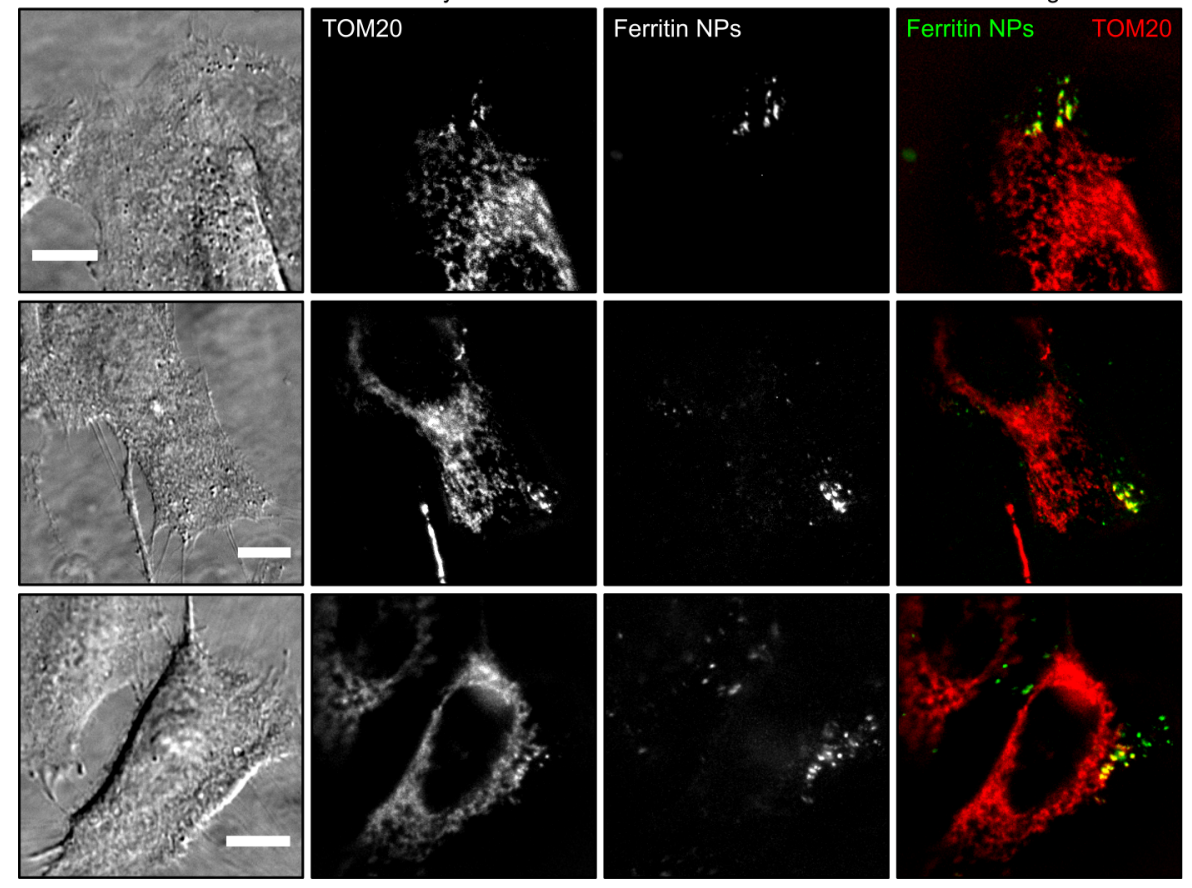

Supplementary Figure 3 | NPs internalized via pinocytosis explore the whole cytoplasm and can target intracellular components

a) QDs behaviour after pinocytic internalization. Top. Transmission image of two HeLa cells. Bottom. Maximum intensity projection (MIP) image of QDs fluorescence from a 2000 frame-long recording. QDs explore the whole cytoplasm of cells (white dotted contours), resulting in a diffuse fluorescence signal in the MIP image, but do not enter into the nuclei (white ovals), which remain dark. Internalization of QDs in living cells via pinocytosis has been achieved successfully more than 100 times. Scale bar represents $10 \mu \mathrm{m}$. 
b) QDs intracellular exploration after passive endosomal internalization. Top. Snapshot of QDs fluorescence. Bottom. MIP of QDs fluorescence from a 500 frame-long recording. In this case, QDs remain trapped inside the endosomes (bright spots in both images) and do not move in the cytosol, resulting in no diffuse fluorescence signal in the MIP image. Passive endosomal internalization of QDs have been repeated twice. Scale bar represents $10 \mu \mathrm{m}$.

c) QDs intracellular exploration after pinocytic internalization. Top. Snapshot of QDs fluorescence. Bottom. MIP of QDs fluorescence from a 500 frame-long recording. Contrarily to when trapped inside endosomes (as in Panel b), after vesicles burst by osmotic shock QDs are released and explore the cytosol, resulting in a diffuse fluorescence signal in the MIP image. Pinocytic internalization of QDs have been successfully done more than 100 times. Scale bar represents $5 \mu \mathrm{m}$.

d) Targeting of ferritin NPs to mitochondria. Examples of HeLa cells expressing the chimeric construct GFPbinder-mCherry-TOM20 (localizing at the mitochondrial membrane) and loaded with ferritin-GFP-NPs using pinocytosis. From left to right: DIC images, mCherry channel, GFP channel, merge (yellow) of mCherry (red) and GFP (green) channels. Co-localization of fluorescence signals shows that ferritin-GFP-NPs could readily target mitochondria, indicating that NPs are dispersed in the cytosol and not encapsulated into pinocytic vesicles. Remarkably, targeting happened on small regions, presumably where vesicles burst, as the result of the very strong affinity (sub-nanomolar $K_{\mathrm{D}}$ ) of the GFP-binder nanobody. The chimeric construct was realized fusing the GFP-binder nanobody and mCherry (used for visualization) to the cytosolic domain of TOM20. Ferritin-GFP-NPs were obtained fusing the heavy chain of ferritin to eGFP. Experiments have been repeated successfully three times. Scale bars represent $10 \mu \mathrm{m}$. 


\section{Supplementary Figure 4}

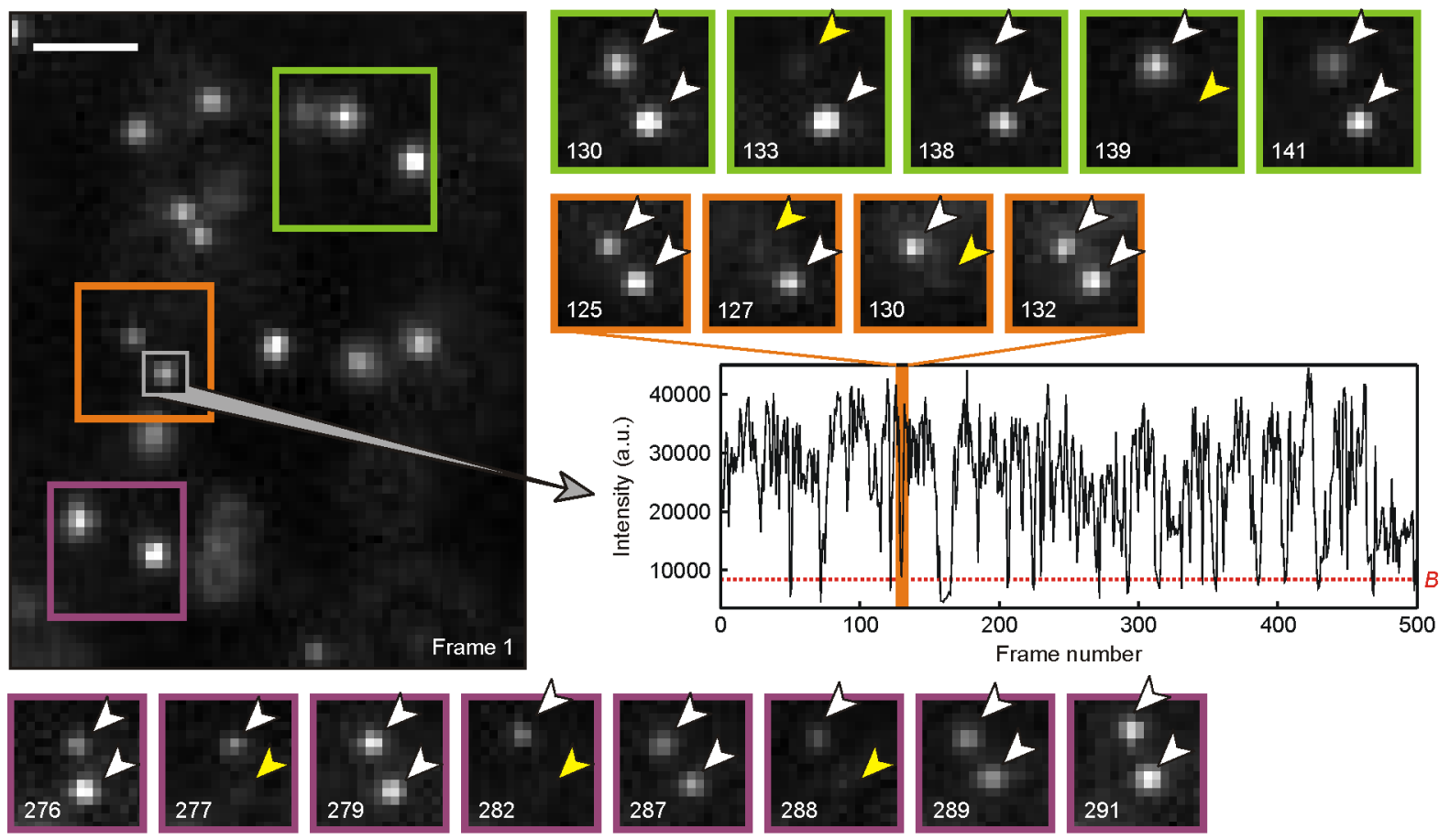

\section{Supplementary Figure 4 | QDs show blinking after pinocytic internalization}

QDs internalized in HeLa cells via pinocytosis clearly showed on-off blinking of the fluorescent signal, a typical signature of individual emitters. This indicates that QDs are mono-dispersed, confirming their escape from pinocytic vesicles and dismissing potential aggregations (especially important in the case of QDs that are, possibly, the probe most prone to aggregate, given their very reactive core not fully screened by the outer functional coating, as evidenced by the results presented in the main text). The large image shows the first frame of Supplementary Video 3 and small crops show sub-sequent frames of the indicated coloured boxes, both for diffusing QDs (green and purple boxes) and for immobile QDs (orange box). In the latter case, the intensity of individual QDs could be easily followed over time integrating the signal of a small region around one NP (grey box). Fluctuations are clearly visible in the corresponding intensity time-course where the red dotted line indicates the background level $B$. White arrowheads point to emitting QDs (on state) while yellow arrowheads to dark QDs (off state). Numbers at the bottom left corner of the coloured boxes indicate the frame from which the image has been extrapolated. Blinking of individual QDs was successfully observed in three independent experiments. Scale bar represents $2 \mu \mathrm{m}$ and applies to all images. 


\section{Supplementary Figure 5}

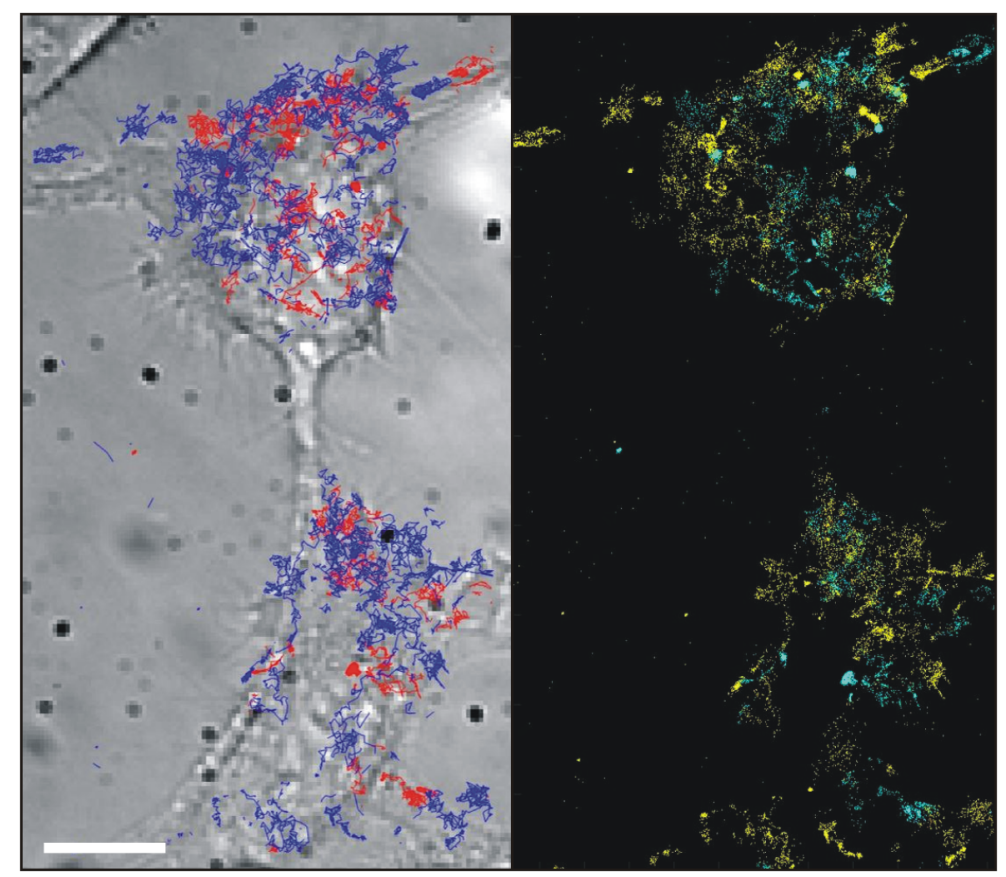

\section{Supplementary Figure 5 | QDs of different colours do not co-localize and diffuse independently after pinocytic internalization}

Left. Composite image showing two HeLa cells (grey scale) and the reconstructed trajectories of two different types of QDs simultaneously internalized via pinocytosis: QDs-605 emitting at $605 \mathrm{~nm}$ (red trajectories) and QDs-PEG- $\mathrm{NH}_{2}$ emitting at $655 \mathrm{~nm}$ (blue trajectories). As visible, most trajectories span relatively large portions of the cells and do not show confinement within regions compatible with the size of pinocytic vesicles (which have a diameter on the order of $2 \mu \mathrm{m}$ ). Right. Maximal projection image showing the detections of QDs-605 (cyan dots) and of QDs-PEG-NH $\mathrm{NH}_{2}$ (yellow dots). No co-localization or correlated dynamics of the two types of QDs has been observed (see also Supplementary Video 4 and 5). Moreover, given the typical numbers of vesicles formed ( 8) and of NPs internalized (between 20 and 50) per cell, the average number of NPs per vesicle is 4 and the probability that all the 8 vesicles formed, on average, in a given cell would be all filled with 4 NPs of the same colour is of the order of $10^{-8}$, which -despite possible- is relatively improbable and further supports the effective release of NPs from pinocytic vesicles. Dual colour experiments have been repeated twice and each time 10 cells have been imaged. Scale bar represents $5 \mu \mathrm{m}$. 


\section{Supplementary Figure 6}
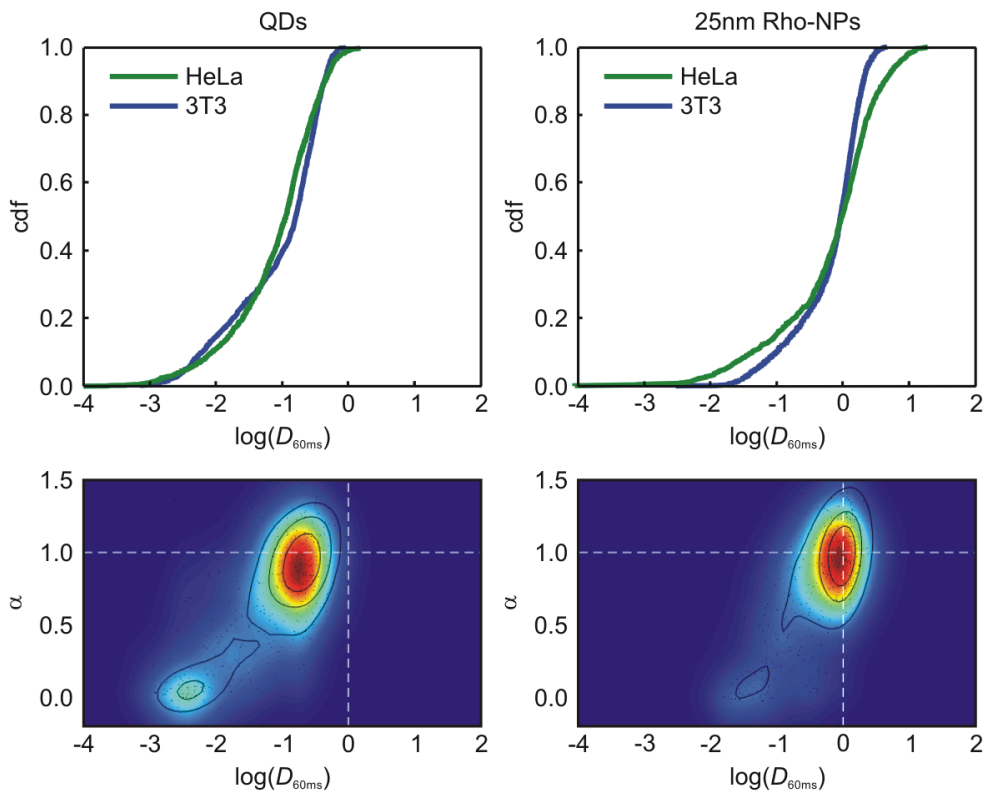

\section{Supplementary Figure 6 | QDs and 25nm Rho-NPs have similar diffusive properties in different mammalian cell lines}

Top. Cumulative distribution function of the logarithm of the instantaneous diffusivity coefficients $D_{60 \mathrm{~ms}}$ for QDs (left) and $25 \mathrm{~nm}$ Rho-NPs (right) in human (cancerous) HeLa cells (green, $N=1696$ trajectories for QDs and $N=1314$ trajectories for Rho-NPs) and in murine (not cancerous) T3T fibroblasts (blue, $N=1316$ trajectories for QDs and $N=2768$ trajectories for Rho-NPs). No substantial quantitative differences emerged from experiments, suggesting uniformity of NPs behaviour in different mammalian cell lines. Bottom. Density plots in the $\alpha-D_{60 \mathrm{~ms}}$ plane for QDs (left, $N=586$ trajectories lasting more than 40 frames, out of 1316 trajectories) and 25nm Rho-NPs (right, $N=698$ trajectories lasting more than 40 frames, out of 2768 trajectories) in $3 \mathrm{~T} 3$ fibroblasts. $D_{60 \mathrm{~ms}}$ is expressed in $\mu \mathrm{m}^{2} \cdot \mathrm{s}^{-1}$. 


\section{Supplementary Figure 7}
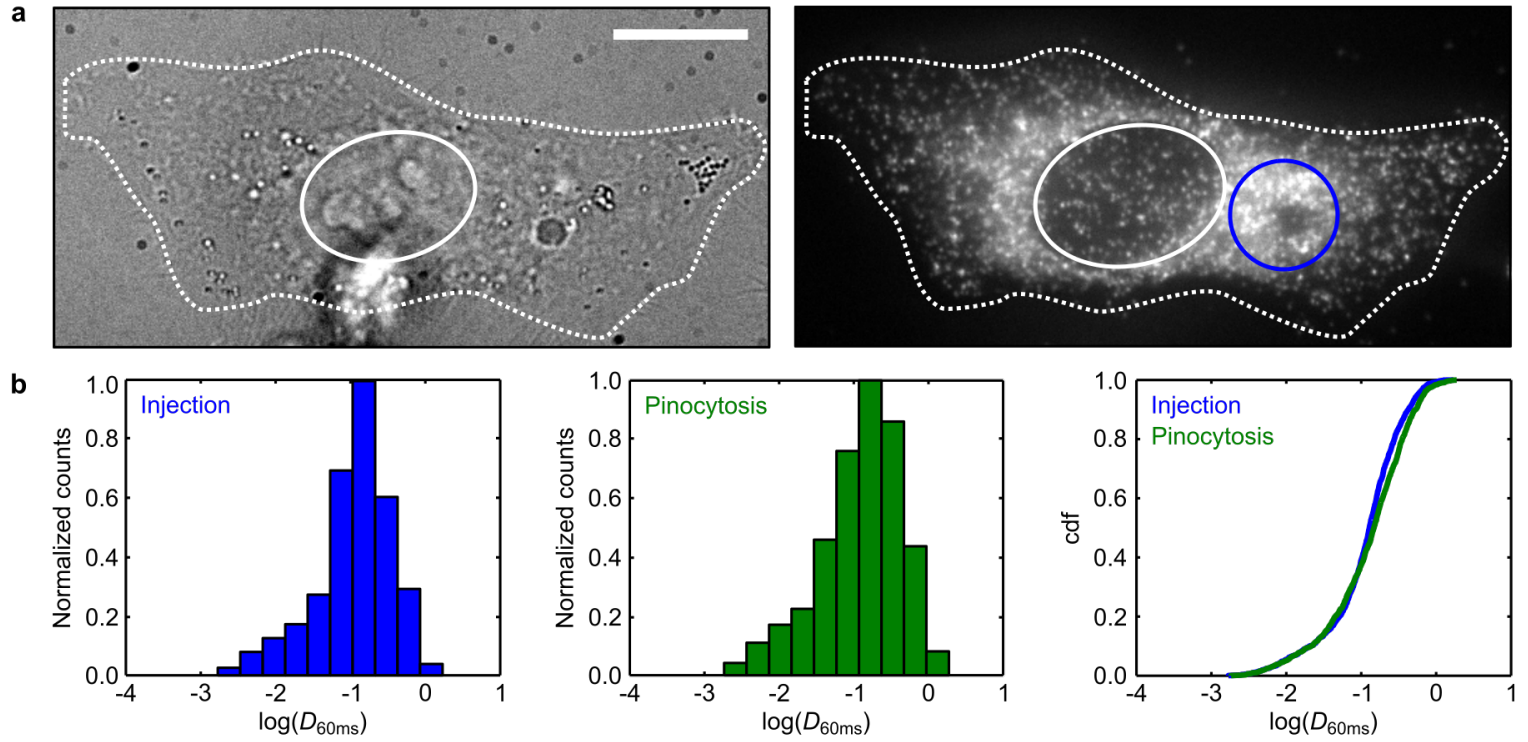

Supplementary Figure 7 | Microinjected NPs show identical diffusive properties than NPs internalized via pinocytosis

a) QDs microinjection. Left. Transmission image of a 3T3 cell (white dotted contour, white oval indicates the cell nucleus). Right. Corresponding fluorescence image of microinjected QDs. The microinjection locus is highlighted by the blue circle and contains a higher density of low mobility NPs. Microinjection experiments were repeated on more than 100 cells in four independent samples. Scale bar indicates $10 \mu \mathrm{m}$.

b) Comparison of NPs mobility for the two internalization methods. From left to right. Normalized distribution of the logarithm of $D_{60 \mathrm{~ms}}$ for QDs microinjected in HeLa cells (blue). Normalized distribution of the logarithm of $D_{60 \mathrm{~ms}}$ for QDs internalized in HeLa cells via pinocytosis (green). Cumulative distribution function of the logarithm of $D_{60 \mathrm{~ms}}$ for QDs microinjected (blue) and internalized via pinocytic loading (green) in HeLa cells. In both representations (histogram and cdf curve), $N=1547$ trajectories of microinjected QDs and $N=1696$ trajectories of QDs internalized via pinocytosis. $D_{60 \mathrm{~ms}}$ is expressed in $\mu \mathrm{m}^{2} \cdot \mathrm{s}^{-1}$. 


\section{Supplementary Figure 8}
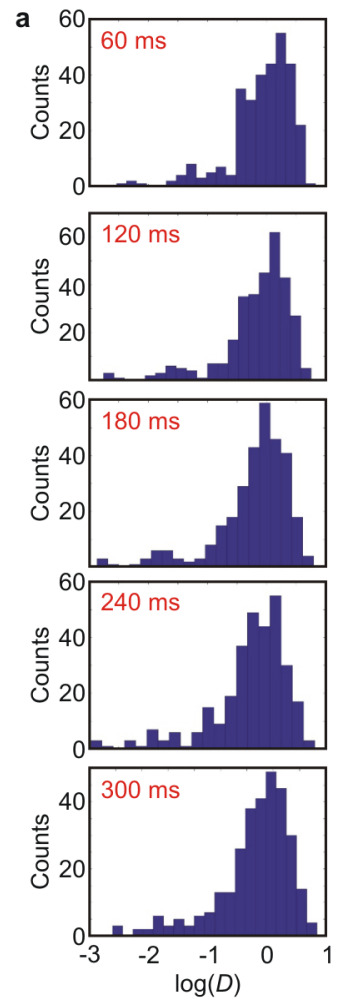
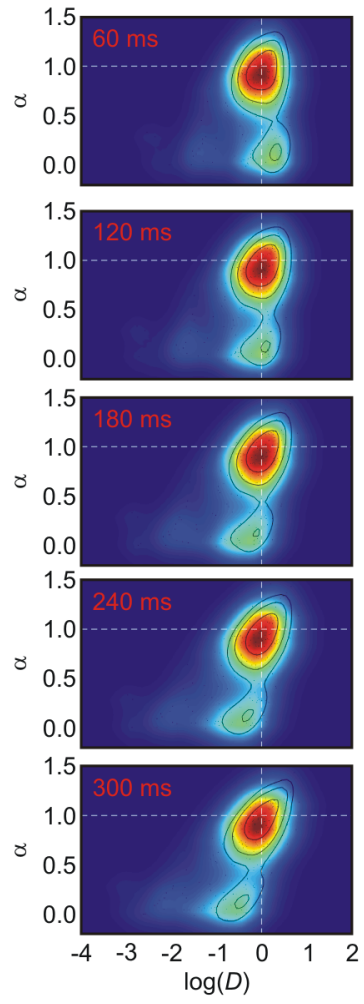
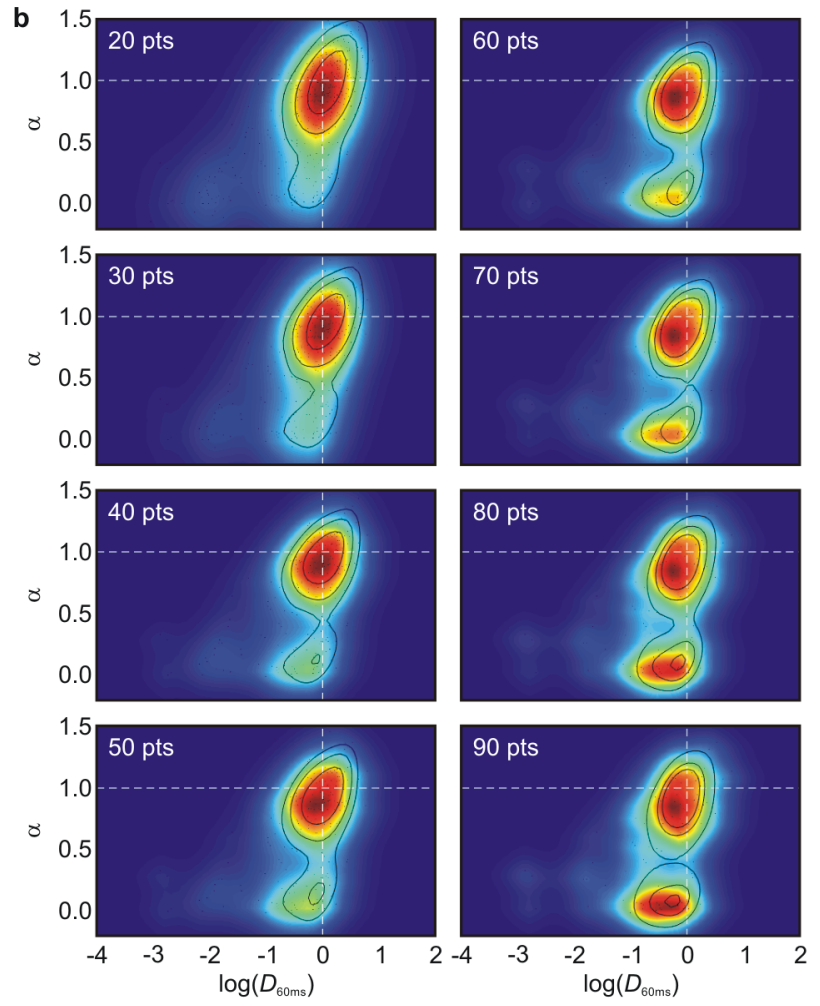

Supplementary Figure $8 \mid \alpha-D_{60} \mathrm{~ms}$ maps are robust with respect to the parameters used for their computation

a) Dependency on the time lag considered for the calculation of the instantaneous diffusivity coefficient $\boldsymbol{D}$. Distributions of the logarithm of $D$ (left) and $\alpha-D$ maps (right) computed considering increasing time lags $(60,120,180,240$, and $300 \mathrm{~ms})$ for the calculation of the instantaneous diffusivity coefficient $D$.

b) Dependency on the minimal trajectory length. $\alpha-D_{60 \mathrm{~ms}}$ maps computed considering an increasing minimal trajectory length (from 20 to 90 time points). Whereas the dependency on the time lag considered for the calculation of the instantaneous diffusivity coefficient $D$ is marginal, the dependency of $\alpha-D_{60 \mathrm{~ms}}$ maps on the minimal trajectory length is more marked. In the results presented in the main text and in Supplementary Fig. 6 and 9, we used a minimal trajectory length of 40 time points to compute the $\alpha-D_{60 \mathrm{~ms}}$ maps. We considered that this choice represented a suitable trade-off between having trajectories long enough to limit data scattering in the maps but still short enough to avoid the strong bias towards severely sub-diffusive trajectories (which will have the tendency to remaining longer in the focal plane generating long trajectories), as highlighted by the results shown in Fig. 3a,b of the main text.

The dataset used for the analysis presented in both panels above is that relative to $25 \mathrm{~nm}$ RhoNPs internalized in HeLa cells via pinocytosis (same dataset used in Fig. 1b and Fig. $2 b$ of the main text, $N=309$ trajectories). $D$ and $D_{60 \mathrm{~ms}}$ are expressed in $\mu \mathrm{m}^{2} \cdot \mathrm{s}^{-1}$. 


\section{Supplementary Figure 9}
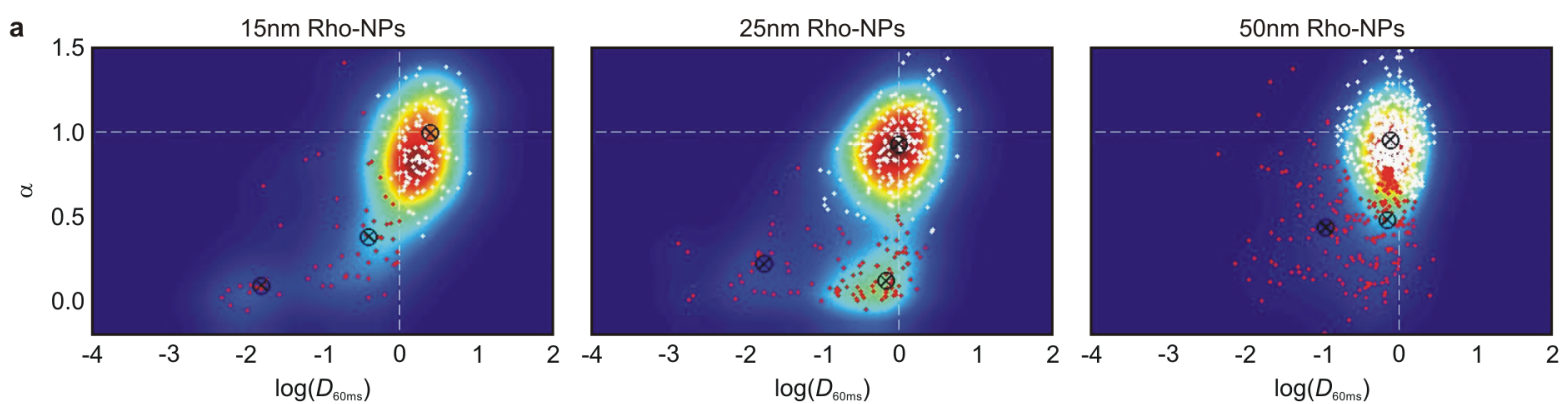

b
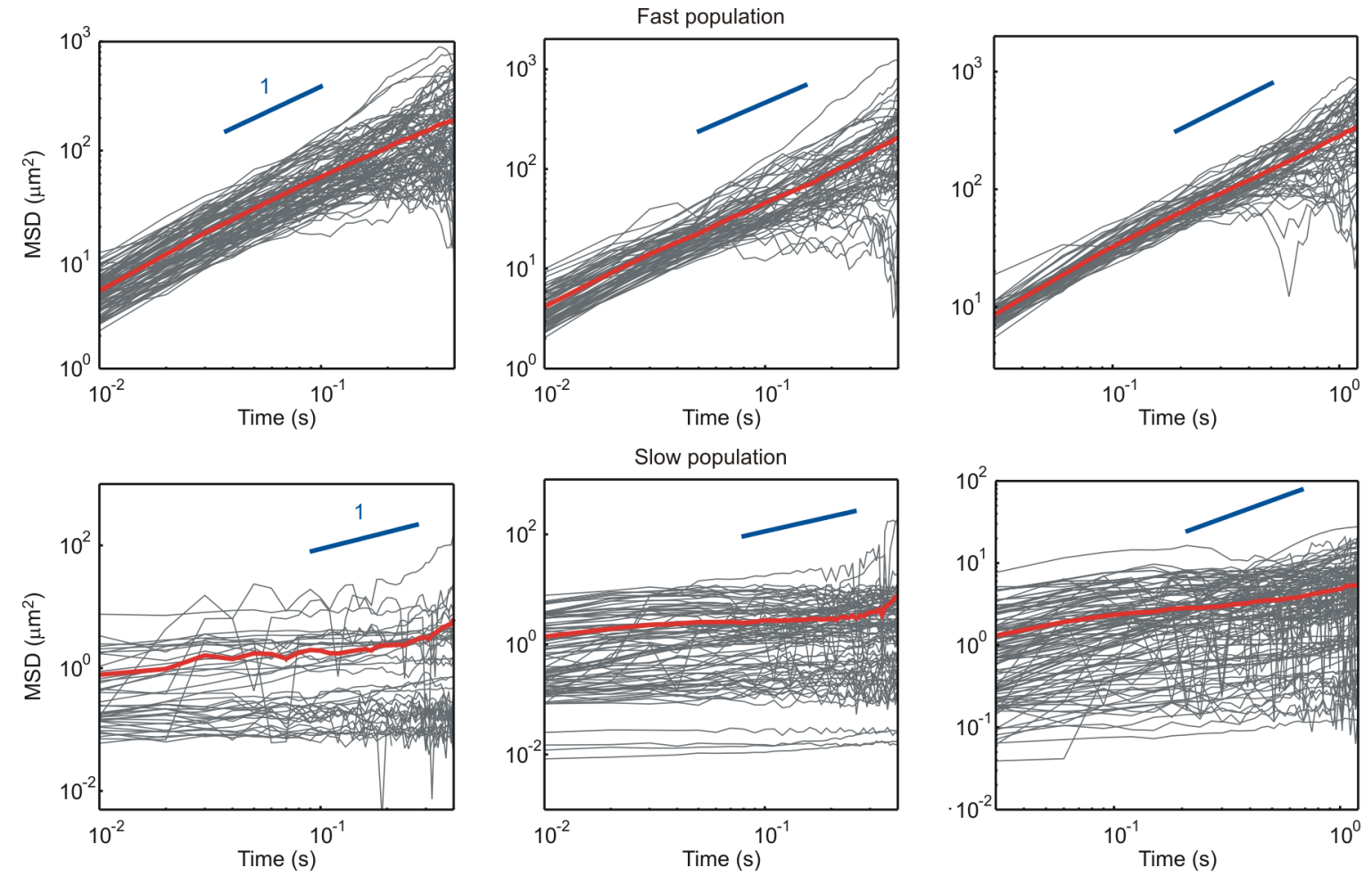

\section{Supplementary Figure 9 | Identification and analysis of Rho-NPs subpopulations}

a) Subpopulation identification via cluster analysis. To discriminate between fast and slow Rho-NPs subpopulations, we used the $k$-mean algorithm in MATLAB to cluster data points in the $\alpha-D_{60 \mathrm{~ms}}$ plane. Two clusters failed to satisfactorily separate the two subpopulations. Thus, we used four clusters and grouped together the two clusters with largest mean $D_{60 \mathrm{~ms}}$ to select trajectories belonging to the fast population (white points in the maps), and the two clusters with the smallest mean $D_{60 \mathrm{~ms}}$ to infer trajectories belonging to the slow population (red points in the maps). Cluster centroids are indicated by black crosses. Relative fractions and mean $D_{60 \mathrm{~ms}}$ values of the two subpopulations are reported in Supplementary Table 1. 75nm Rho-NPs being mostly immobile, or very slowly diffusing, were not considered for the subpopulation analysis. The number of trajectories in the $\alpha-D_{60 \mathrm{~ms}}$ maps is: $N=186$ for $15 \mathrm{~nm}$ Rho-NPs, $N=309$ for $25 \mathrm{~nm}$ Rho-NPs, and $N=837$ for $50 \mathrm{~nm}$ Rho-NPs. $D_{60 \mathrm{~ms}}$ is expressed in $\mu \mathrm{m}^{2} \cdot \mathrm{s}^{-1}$. 
b) MSD curves of fast and slow Rho-NPs subpopulations. Individual time-averaged MSDs (grey curves), in log-log scale, for the indicated Rho-NPs in HeLa cells after classification into fast (top row) and slow (bottom row) subpopulation with the cluster analysis. Blue lines have a slope of 1 (Brownian diffusion), red lines are ensemble averages. The fraction of trajectories in each of the two subpopulations is reported in Supplementary Table 1. 


\section{Supplementary Figure 10}

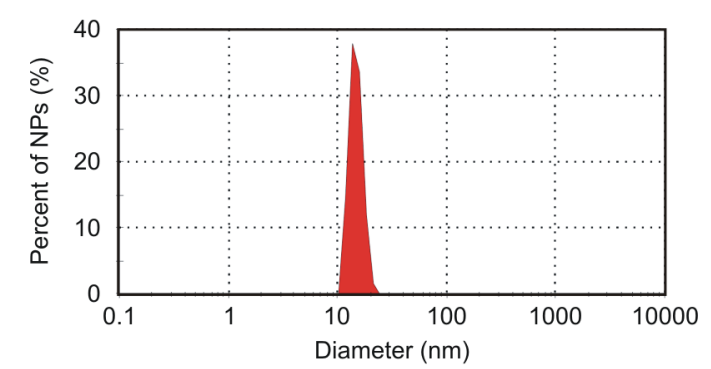

c

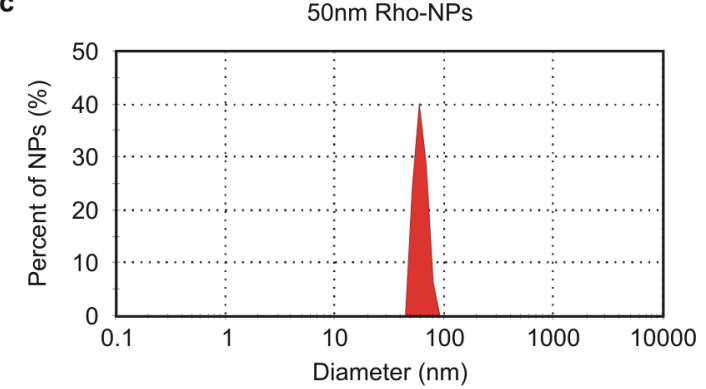

b

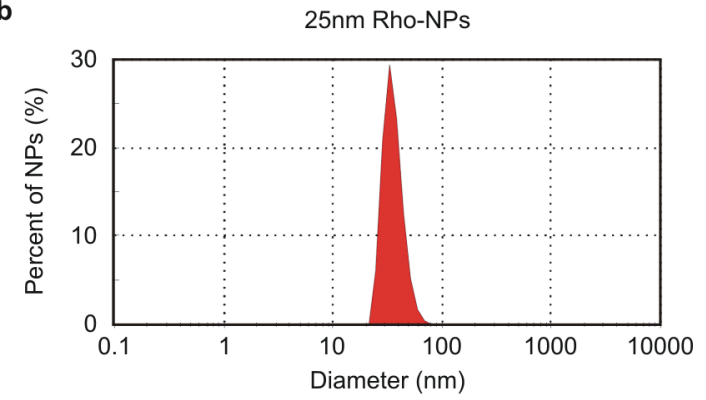

d

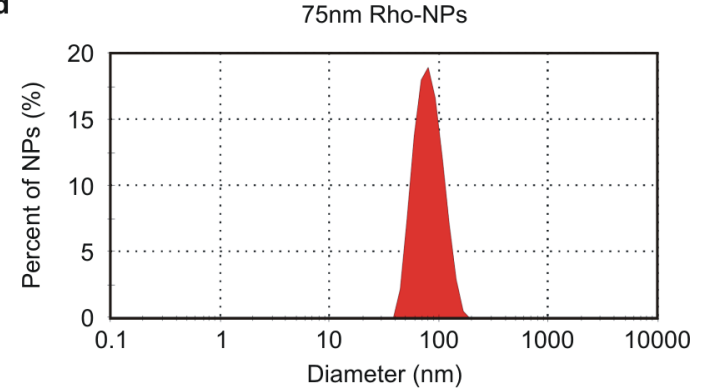

\section{Supplementary Figure 10 | Size distribution of Rho-NPs}

Distribution of Rho-NPs diameter measured by dynamic light scattering (courtesy of micromod Partikeltechnologie $\mathrm{GmbH}$ ).

a) 15nm (plain $\mathbf{N H}_{2}$ ) Rho-NPs (product no. 30-01-151, lot no. 0030930-00) have a size of $(15 \pm 2)$ nm. $N=8 \times 10^{6}$ particles.

b) 25nm (streptavidin) Rho-NPs (product no. 30-19-251, lot no. 0451130-00) have a size of $(35 \pm 8) \mathrm{nm} . N=12.1 \times 10^{6}$ particles.

c) 50nm (streptavidin) Rho-NPs (product no. 30-19-501, lot no. 0101230-1) have a size of $(56 \pm 9) \mathrm{nm} . N=7.5 \times 10^{6}$ particles.

d) 75nm (streptavidin) Rho-NPs (product no. 30-19-751, lot no. 0211230-00) have a size of $(77 \pm 14) \mathrm{nm} . N=27.7 \times 10^{6}$ particles.

Reported NPs size values are mean \pm SEM. 


\section{Supplementary Figure 11}

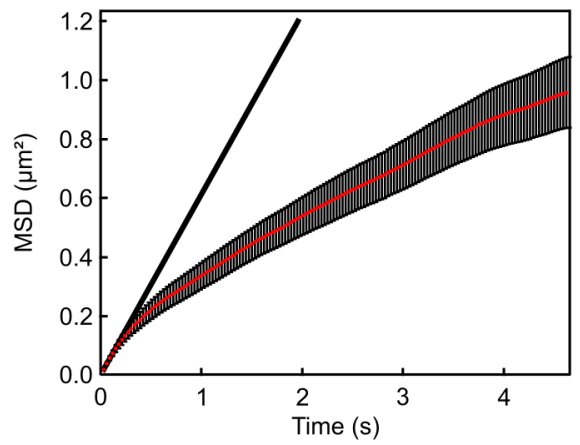

\section{Supplementary Figure 11 | The ensemble-averaged MSD of QDs is sub-linear}

The graph shows (in linear scale) the ensemble average of all individual, temporally-averaged MSDs, $\left\langle\left\langle r^{2}\right\rangle_{\mathrm{T}}\right\rangle_{\mathrm{E}}$, of QDs internalized in HeLa cells via pinocytosis $(N=1696$ trajectories, same dataset than in Fig. 1c and Fig. 3a of the main text). The sub-linearity is clearly shown by the difference between the MSD curve obtained for QDs (red) and the straight line (black) corresponding to the MSD of a Brownian particle with a diffusion coefficient equal to the instantaneous diffusivity coefficient of QDs, $D_{60 \mathrm{~ms}}=(0.15 \pm 0.01) \mu \mathrm{m}^{2} \cdot \mathrm{s}^{-1}$. Error bars represent SEM. 


\section{Supplementary Figure 12}

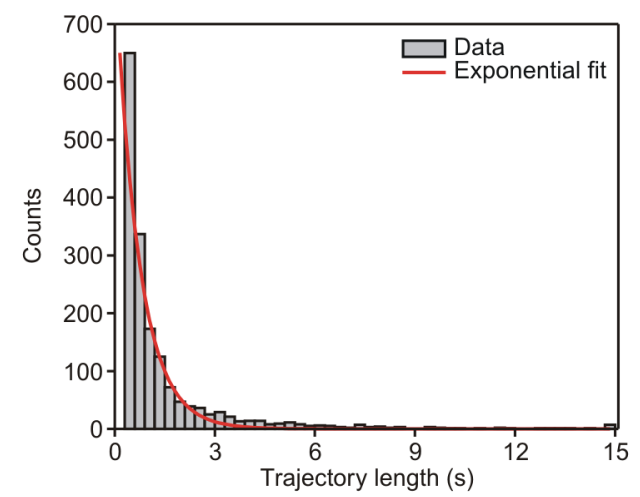

\section{Supplementary Figure 12 | Trajectory length follows an exponential distribution}

The histogram (grey columns) shows the trajectory length distribution relative to QDs internalized in HeLa cells via pinocytosis. Red line represents the best fit to data using a monoexponential decay function $(\tau=0.7 \pm 0.1 \mathrm{~s}) . N=1696$ trajectories. 


\section{Supplementary Figure 13}
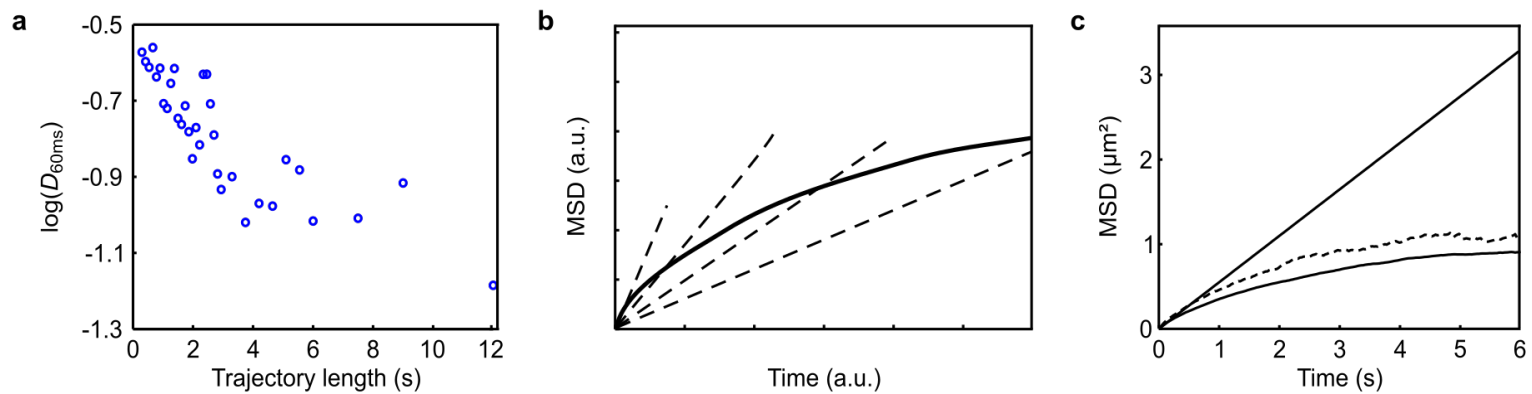

\section{Supplementary Figure 13 | Averaging of trajectories with different lengths results in artefactual sub-diffusivity}

a) Dependence of $\boldsymbol{D}_{60 \mathrm{~ms}}$ on trajectory length. Scatter plot of the logarithm of the instantaneous diffusivity coefficient $D_{60 \mathrm{~ms}}$ as a function of the trajectory length for QDs internalized via pinocytosis in HeLa cells $(N=1696$ trajectories, binned into 34 intervals of different trajectory lengths). Data clearly indicate a negative correlation between $D_{60 \mathrm{~ms}}$ and the trajectory length: the longer the trajectory the smaller the instantaneous diffusivity coefficient. As a consequence, length has a pronounced effect in trajectory analysis (as shown for $25 \mathrm{~nm}$ Rho-NPs in Supplementary Fig. 8b) and, if not properly taken into account, it may profoundly affect the analysis of the ensemble behaviour of NPs (see Fig. 3a,b of the main text). Furthermore, at long time scales, the bias towards slow (and severely sub-diffusive) trajectories reflects into an accentuated, or even artefactual, sub-diffusive ensemble behaviour of NPs (see Panels b and c). $D_{60 \mathrm{~ms}}$ is expressed in $\mu \mathrm{m}^{2} \cdot \mathrm{s}^{-1}$.

b) Artefactual sub-diffusivity of averaged MSDs. The graph shows four fictive linear MSDs curves (dashed lines). They represent theoretical MSDs of four Brownian particles with four different diffusion coefficients. As in experiments, rapidly diffusing particles leave quickly the observation volume, resulting in short trajectories and MSD curves extended over few time lags. Inversely, slowly diffusing particles have longer trajectories and MSD curves. Averaging of such not homogeneous, although linear, MSD curves gives rise to an artefactual sublinear ensemble MSD (solid line).

c) Ensemble averaging of Brownian trajectories -artificially generated from QDs datashows sub-diffusion. For each recorded trajectory (of QDs in HeLa cells), we extracted two parameters: the trajectory length $L$ and the instantaneous diffusivity coefficient $D_{60 \mathrm{~ms}}$. From these two parameters, we build a set of linear MSD curves, each of them having duration $L$ and slope determined by $D_{60 \mathrm{~ms}}$. Remarkably, such an artificial set of perfectly Brownian MSDs resulted in a sub-diffusive ensemble-averaged MSD (dashed line), with approximately the same shape of the experimental one (solid line, same as red curve in Supplementary Fig. 11) and markedly deviating from Brownian diffusion (solid straight line).

Although rough, the calculations reported above (Panels b and c) highlight how sub-diffusive behaviour can emerge as an artefact inherent to the averaging over a set of purely Brownian trajectories with different length. 


\section{Supplementary Figure 14}

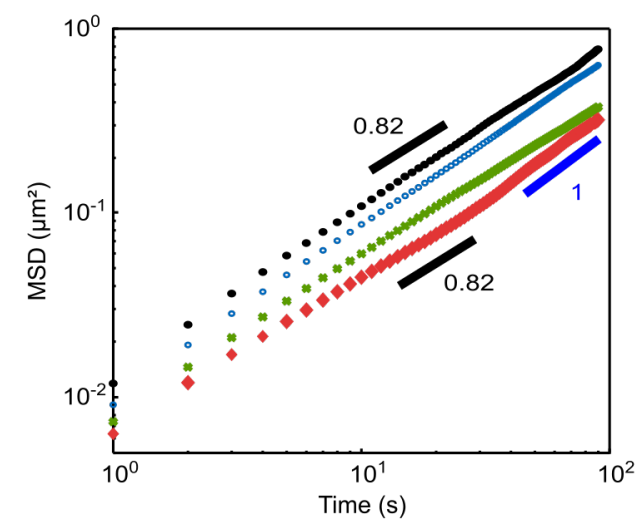

\section{Supplementary Figure 14 | Drug treatments have minor effects on QDs diffusive behaviour}

The figure shows ensemble-averaged MSD curves of QDs in log-log scale, computed considering trajectories lasting more than 90 frames, obtained in HeLa cells after different drug treatments: basal conditions -untreated cells- (black), Nocodazole $(0.1 \mu \mathrm{M})$ treated cells (blue), Latrunculin-A $(0.1 \mu \mathrm{M})$ treated cells (green), Brefeldin-A $(5 \mu \mathrm{M})$ treated cells (red). Despite slight variations in diffusivity (vertical spread of the curves), all MSDs are linear (in log-log scale) and, remarkably, they all have the same slope (0.82), pointing to an intrinsic anomalous behaviour of QDs, very mildly or not affected at all by the drug treatments used. In fact, only for Brefeldin-A treatment, and only at a long timescale, we could observe a deviation of the value of the MSD slope (from 0.82 to 1). QDs were internalised in HeLa cells via pinocytosis. Basal conditions: $N=216$ trajectories lasting more than 90 frames, out of 1696 trajectories. Nocodazole treated cells: $N=486$ trajectories out of 2189 trajectories. Latrunculin-A treated cells: $N=312$ trajectories out of 1126 trajectories. Brefeldin-A treated cells: $N=220$ trajectories out of 1101 trajectories. 


\section{Supplementary Figure 15}

a
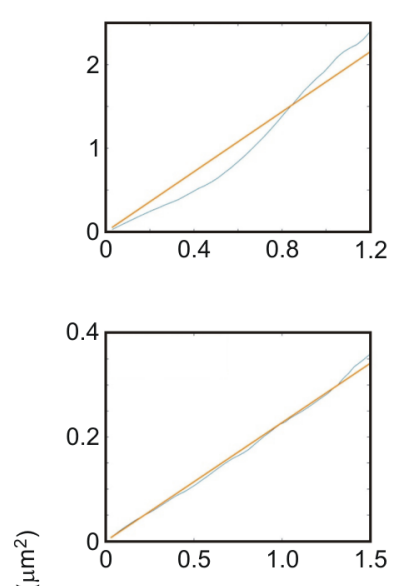

की
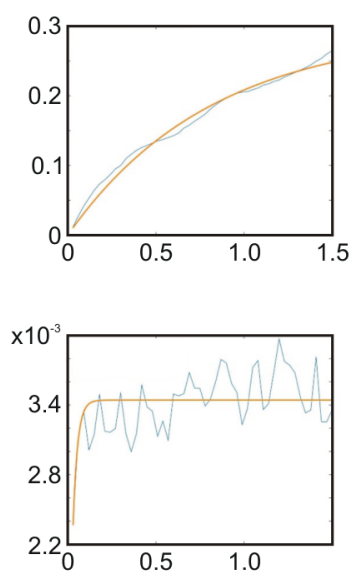

b
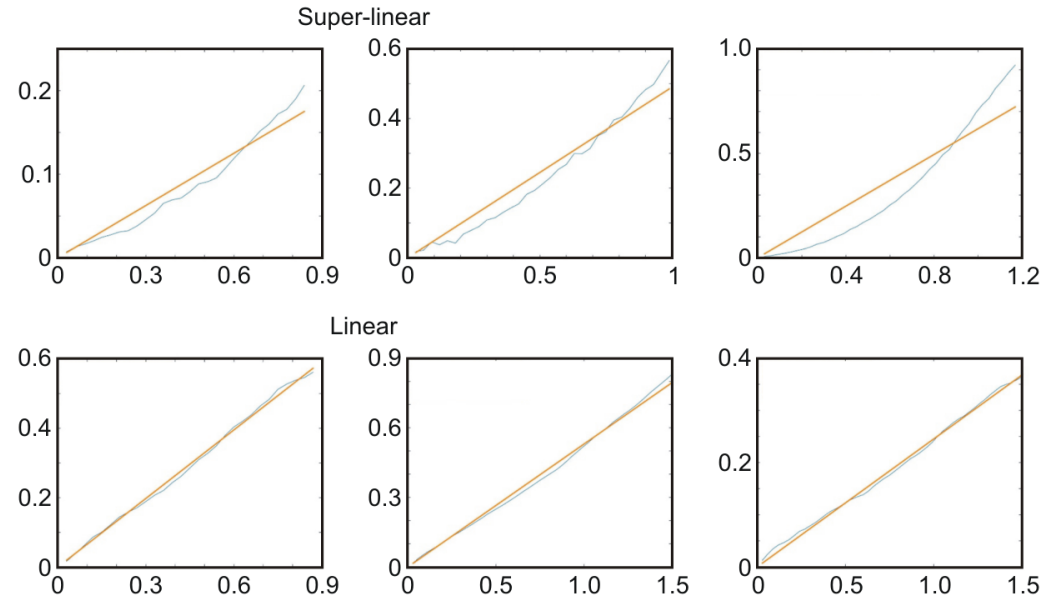

Linear
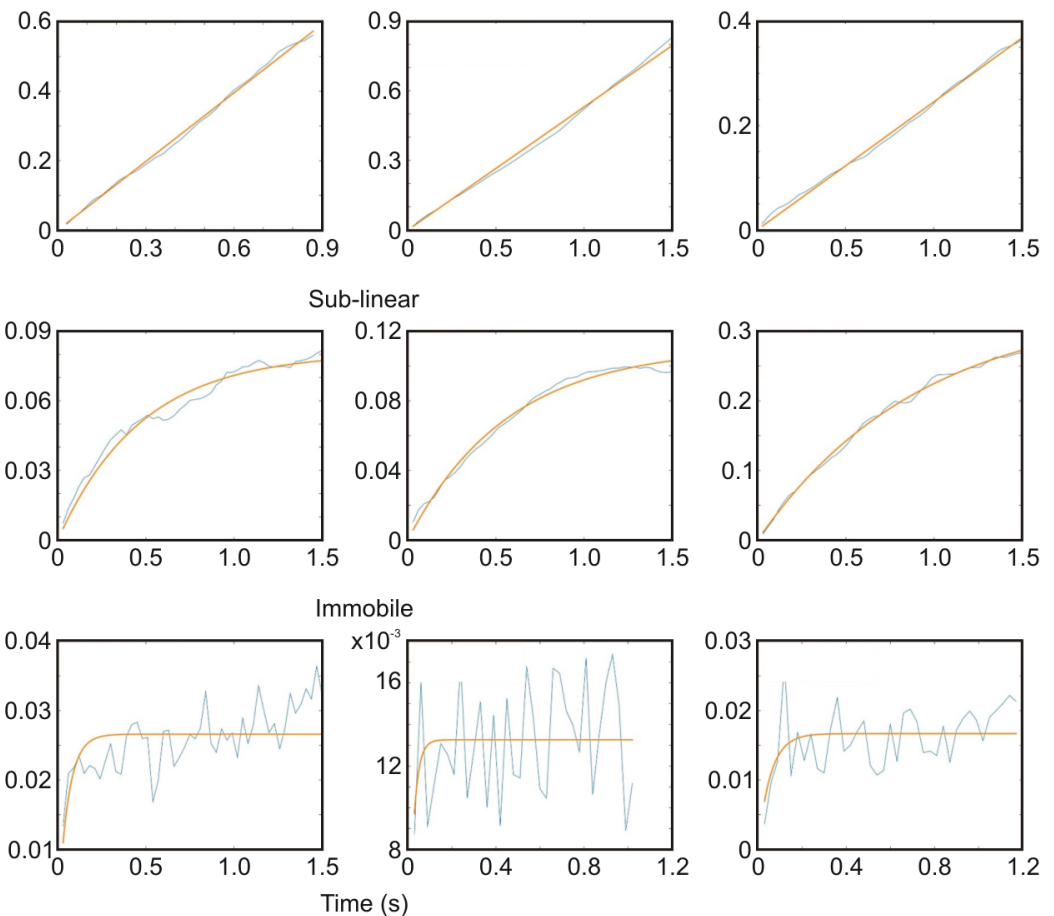

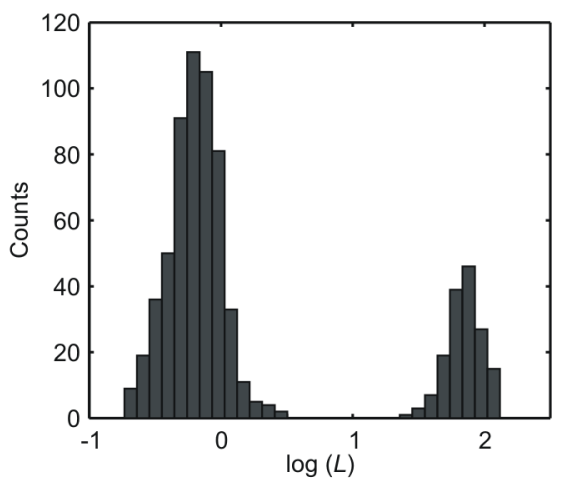

Supplementary Figure 15 | QDs mobility shows no strong evidence of confined diffusion

a) Confined diffusion fits of experimental MSD curves. Examples of time-averaged MSD curves, lasting more than 30 frames, obtained from QDs internalised in (untreated) HeLa cells via pinocytosis. MSD curves (cyan lines) have been fitted with a confined diffusion model (orange lines) using the formula: $\operatorname{MSD}(t)=L^{2} \cdot\left(1-\exp \left(-12 D t / L^{2}\right)\right)$, where $L$ represents 
the size of the confinement box and $D$ the diffusion coefficient. Despite MSD curves are expected to be sub-linear in the case of confined diffusion, we found also linear -and even super-linear- MSDs. Overall, four different MSD categories emerged: super-linear, linear, sublinear, and immobile (examples of each are shown in the four rows from top to bottom, respectively).

b) Box size distribution from confined diffusion fits in basal conditions. When we globally analysed the behaviour of QDs in HeLa cells, we obtained a bimodal distribution of the logarithm of the box size $L$ (expressed in $\mu \mathrm{m}, N=685$ trajectories), which spanned more than three orders of magnitude. The highest values of $L$ correspond to trajectories with linear or super-linear MSDs (as in the first two rows in Panel a) that, when fitted with a confined diffusion model (even if the residuals of the fit are small), are basically fitted by straight lines, resulting in unrealistic box sizes and no physical significance of the parameter $L$ (i.e., $L$ larger than the cell). Conversely, the second population with smaller values of $L(77 \%$ of all trajectories) corresponds to trajectories (as in the third row in Panel a) fitted with a physically plausible box size, with a mean $L$ value of $630 \mathrm{~nm}$. Nevertheless, numerical simulations showed that a similar degree of confinement (box size) is found even for unrestricted, Brownian trajectories. In fact, due to the intrinsic variability of MSD curves, even a large fraction $(60 \%)$ of simulated trajectories, of particles diffusing in free space, could be fitted with a confined diffusion model, resulting into a mean box size of $850 \mathrm{~nm}$ (see Supplementary Table 2 and Methods for details of simulations). This value does not differ significantly from the one found experimentally and, in our view, indicates that the (putative) box size found (for QDs) is mostly a consequence of the limited temporal window of observation of QDs dynamics. Furthermore, in no case (except for completely immobile QDs, as in the last row in Panel a) we could observe a clear plateau in the MSD of individual particles, the most unambiguous signature of confined diffusion. Finally, the different drug treatments tested did not provoke substantial changes in the estimates of the mean box size values nor in the percentage of confined trajectories while, contrarily to plausible expectations, they led to reduced box sizes and to increased percentages of confined trajectories (see Supplementary Table 2). We conclude that, overall, there is no strong evidence of confined diffusion for QDs. 


\section{Supplementary Figure 16}
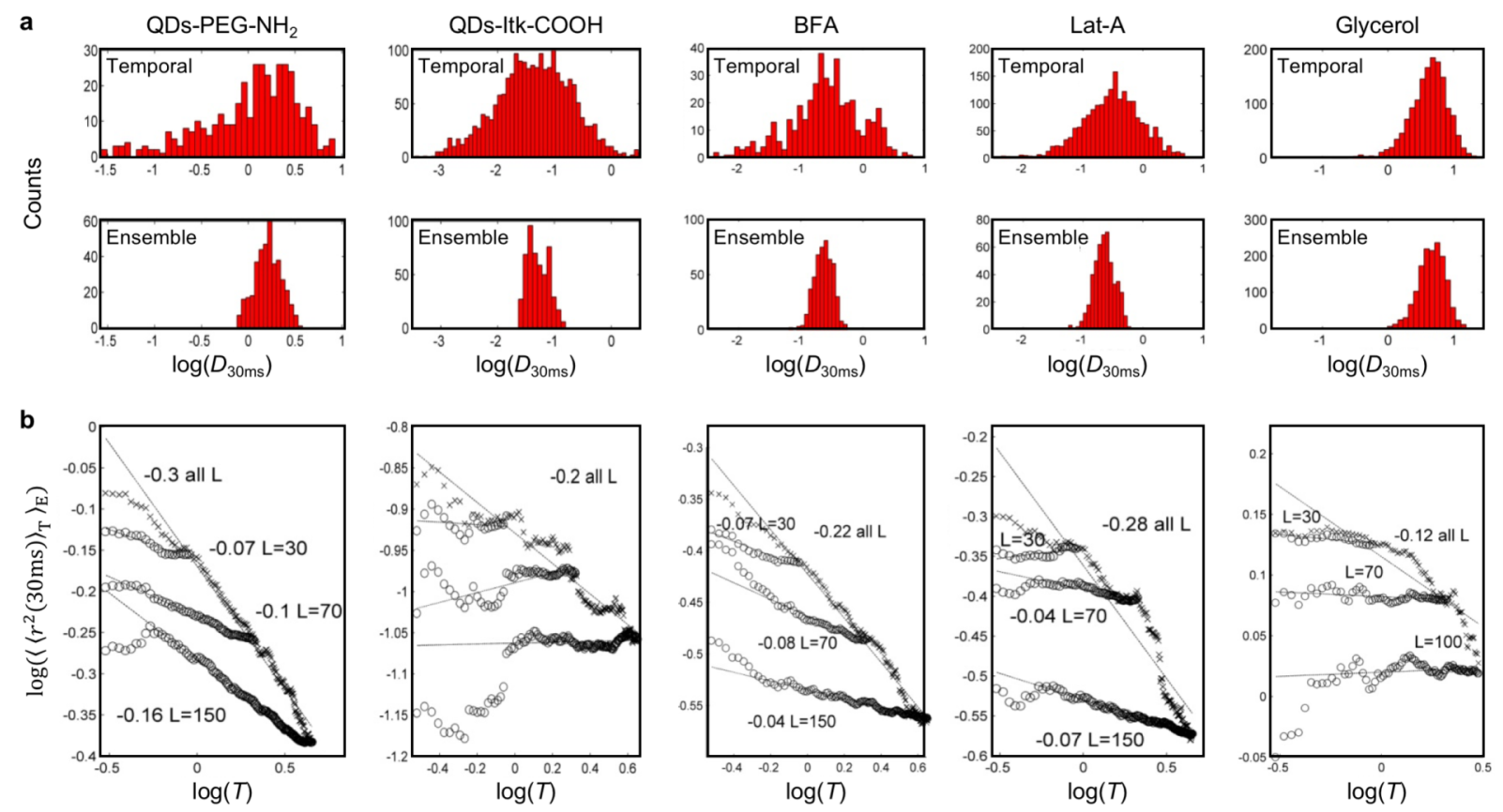

$\left\langle\left\langle r^{2}\left(t_{\operatorname{lag}}, T \mid L\right)\right\rangle_{\mathrm{T} \mid \mathrm{L}}\right\rangle_{\mathrm{E}} \propto T^{\gamma-1} \quad \gamma=f(L)$

\section{Supplementary Figure 16 | QDs trajectories show ergodicity breaking and aging}

a) Ergodicity breaking. Temporal (top) versus ensemble (bottom) average distributions of the instantaneous diffusivity coefficients (calculated at $30 \mathrm{~ms}$ ) for different probes (QDs-PEG- $\mathrm{NH}_{2}$ and QDs-Itk-COOH) in basal conditions (untreated cells), and for QDs-PEG-NH 2 in cells treated with Brefeldin-A (BFA) or Latrunculin-A (Lat-A) or dispersed in glycerol $(80 \% \mathrm{v} / \mathrm{v}$ in water). Ergodicity breaking is shown by the different broadness of the temporal and ensemble distributions observed in all cases but for glycerol. Experiments, except for glycerol were performed in HeLa cells and QDs internalized via pinocytosis. $D_{30 \mathrm{~ms}}$ is expressed in $\mu \mathrm{m}^{2} \cdot \mathrm{s}^{-1}$. b) Aging. Ensemble average of time-averaged mean square displacements (calculated using the formula reported at the bottom and expressed in $\mu \mathrm{m}^{2}$ ) for a lag time of $30 \mathrm{~ms}$ as a function of the truncation time $T$ applied to the trajectories, for the above-mentioned probes and conditions. For QD-PEG-NH $\mathrm{NH}_{2}$ in basal conditions, as well as after Latrunculin-A and Brefeldin-A treatments, we observed aging in trajectories, namely a power law dependency on the truncation time $T$. As indicated in the graphs, the exponent of the power law depends on the length $L$ of the trajectories considered in the analysis. For "naked" QDs (QD-Itk-COOH), we did not observe aging, probably because of the extremely long binding times and the consequent low number of moving particles. Remarkably, as expected, QDs diffusing in glycerol did not show any sign of aging, on top of no sign of ergodicity breaking.

In both panels, the number $N$ of trajectories included in the analysis is equal to 1696 for QDs-PEG-NH $\mathrm{NH}_{2}$ in untreated cells, 915 for QDs-Itk-COOH in untreated cells, 1101 for QDs-PEG-NH $\mathrm{NH}_{2}$ in Brefeldin-A treated cells, 1126 for QDs-PEG-NH $\mathrm{NH}_{2}$ in Latrunculin-A treated cells, and 5118 for QDs-PEG-NH 2 in $80 \%(\mathrm{v} / \mathrm{v})$ glycerol. 


\section{Supplementary Figure 17}
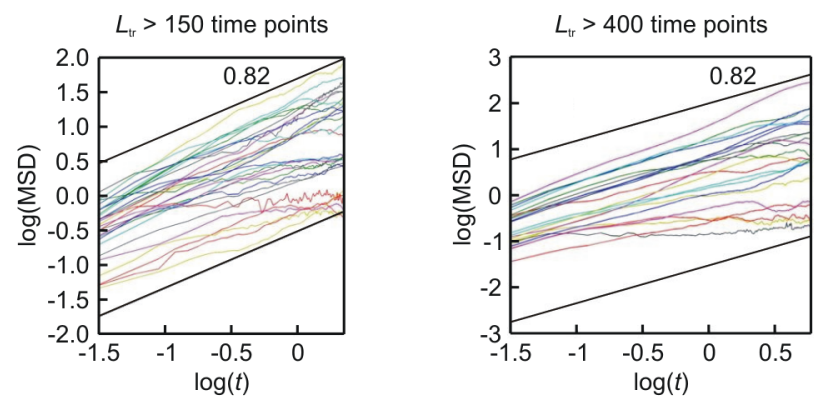

Supplementary Figure 17 | Time-averaged MSD curves of QDs show sub-diffusive behaviour

The figure shows several different examples of individual time-averaged MSD curves, in $\log -\log$ scale, for QDs trajectory with length $\left(L_{\mathrm{tr}}\right)$ lasting more 150 (left) and 400 (right) time points. Straight black lines represent the expected MSD for anomalous diffusion with an exponent $\alpha$ equal to 0.82. QDs (QDs-PEG- $\mathrm{NH}_{2}$ ) were internalised in (untreated) HeLa cells via pinocytosis. Experiments have been repeated three times with similar results. MSD is expressed in $\mu \mathrm{m}^{2}$ and $t$ in seconds. 


\section{Supplementary Table 1}

\begin{tabular}{|c|c|c|c|c|c|c|c|}
\hline \multirow{2}{*}{$\begin{array}{l}\text { Rho-NPs } \\
\text { diameter }\end{array}$} & \multicolumn{3}{|c|}{ Slow population } & \multicolumn{3}{|c|}{ Fast population } & \multirow[b]{2}{*}{$\eta_{\text {cyto }} / \eta_{\mathrm{H}_{2} \mathrm{O}}$} \\
\hline & Fraction & $\begin{array}{c}D_{60 \mathrm{~ms}} \\
\left(\mu \mathrm{m}^{2} \cdot \mathrm{s}^{-1}\right)\end{array}$ & $\underset{\left(\mu \mathbf{m}^{2} \cdot \mathbf{s}^{-1}\right)}{\text { SEM }}$ & Fraction & $\underset{\left(\mu \mathrm{m}^{2} \cdot \mathbf{s}^{-1}\right)}{D_{60 \mathrm{~ms}}}$ & $\underset{\left(\mu \mathbf{m}^{2} \cdot \mathbf{s}^{-1}\right)}{\text { SEM }}$ & \\
\hline $15 \mathrm{~nm}$ & 0.30 & 0.33 & 0.04 & 0.70 & 2.50 & 0.14 & 13.6 \\
\hline $25 \mathrm{~nm}$ & 0.33 & 0.51 & 0.05 & 0.67 & 1.34 & 0.07 & 15.2 \\
\hline $50 \mathrm{~nm}$ & 0.29 & 0.54 & 0.03 & 0.71 & 0.94 & 0.02 & 10.9 \\
\hline
\end{tabular}

\section{Supplementary Table 1 | Mobility of 15nm, 25nm, and 50nm Rho-NPs}

The Table reports the mobility parameters of the fast and slow population of Rho-NPs in HeLa cells obtained using the $k$-mean algorithm for subpopulation identification and separation (see Supplementary Fig. 9a and Methods for details).

Fraction indicates the subpopulation abundance, $D_{60 \mathrm{~ms}}$ the mean diffusivity value and SEM the associated standard error of the mean.

$\eta_{\text {cyto }}$ was calculated using the Stokes-Einstein equation: $D_{60 \mathrm{~ms}}=k_{\mathrm{B}} T / 6 \pi \eta_{\text {cyto }} R$, where $k_{\mathrm{B}}$ is the Boltzmann constant, $R$ the radius of the NPs, and considering the $D_{60 \mathrm{~ms}}$ value relative to the fast population of NPs. In the calculation, the dynamic viscosity of water $\left(\eta_{\mathrm{H}_{2} \mathrm{O}}\right)$ at $37^{\circ} \mathrm{C}$ has been assumed to be equal to $6.9 \times 10^{-4} \mathrm{~N} \cdot \mathrm{s} \cdot \mathrm{m}^{-2}$.

The total number of trajectories considered in the analysis is $N=186$ for $15 \mathrm{~nm}$ Rho-NPs, $N=309$ for $25 \mathrm{~nm}$ Rho-NPs, and $N=837$ for 50nm Rho-NPs. 


\section{Supplementary Table 2}

\begin{tabular}{|c|c|c|c|c|c|}
\cline { 2 - 5 } \multicolumn{1}{c|}{} & $\begin{array}{c}\text { Basal } \\
\text { conditions }\end{array}$ & $\begin{array}{c}\text { Lat-A } \\
\mathbf{( 1 0 0} \mathbf{~ n M})\end{array}$ & $\begin{array}{c}\text { Nocodazole } \\
\mathbf{( 1 0 0} \mathbf{n M})\end{array}$ & $\begin{array}{c}\text { BFA } \\
\mathbf{( 5} \boldsymbol{\mu M})\end{array}$ & $\begin{array}{c}\text { Simulated } \\
\text { Brownian }\end{array}$ \\
\hline $\begin{array}{c}\text { Percentage of } \\
\text { confined trajectories }\end{array}$ & $77 \%$ & $83 \%$ & $75 \%$ & $86 \%$ & $60 \%$ \\
\hline Mean box size & $630 \mathrm{~nm}$ & $500 \mathrm{~nm}$ & $600 \mathrm{~nm}$ & $478 \mathrm{~nm}$ & $830 \mathrm{~nm}$ \\
\hline
\end{tabular}

\section{Supplementary Table 2 | Effects of drug treatments on QDs confinement}

The percentage of confined trajectories has been estimated from the relative amplitude of the two peaks in the bimodal distribution of the confinement box size (see Supplementary Fig. 15b). Mean box size represents the mean value of the peak with the smaller (and physically plausible) box size dimension (left peak in Supplementary Fig. 15b).

The results reported in the Table refer to QDs (QDs-PEG-NH 2$)$ internalized in HeLa cells via pinocytosis in basal condition ( $N=1696$ trajectories) and after different drug treatments ( $N=1126$ trajectories for Lat-A; $N=2189$ trajectories for Nocodazole; and $N=1101$ trajectories for BFA), as well as for simulated Brownian trajectories $(N=4000$ simulated trajectories). Lat-A stands for Latrunculin-A and BFA for Brefeldin-A.

As briefly mentioned in the caption of Supplementary Fig. 15b, if confinement would be effectively present and related to compartmentalization of the cytoplasm, treatments with drugs that affect the cytoplasm organization should induce a significant decrease of the fraction of confined QDs and/or an increase in the mean box size. Nevertheless, any change at all was found for cells treated with Nocodazole, a microtubule-depolymerizing drug. Treatment with Latruculin-A or Brefeldin-A, which respectively disrupts the actin cytoskeleton and the Golgi apparatus, inversely to expectations, caused a slight decrease of the box size and a mild increase of the fraction of confined trajectories. Moreover, analysis of simulated trajectories showed that a large fraction $(60 \%)$ of time-averaged MSDs of freely diffusing trajectories, with similar characteristics as those observed for QDs data (see Methods for details), could be as well fitted with a confined diffusion model, as a consequence of the intrinsic variability of Brownian motion and MSD curves, resulting in a mean box size of $850 \mathrm{~nm}$.

Altogether, this suggests that the putative confinement found for QDs principally reflects the limited extent of the temporal window of observation of their dynamics and not real confined diffusion. 


\section{Supplementary Table 3}

\begin{tabular}{|c|c|c|c|}
\hline NPs & $\begin{array}{c}\text { Zeta potential } \\
\mathbf{( m V )}\end{array}$ & $\begin{array}{c}\text { Immobile fraction } \\
\mathbf{( \% )}\end{array}$ & \multicolumn{2}{c|}{$\begin{array}{c}\boldsymbol{D}_{\mathbf{6 0 m s}} \\
\left(\boldsymbol{\mu m}^{\mathbf{2}} \cdot \mathbf{s}^{-1}\right)\end{array}$} \\
\hline QDs-PEG-NH & -6.5 & $36 \pm 15$ & 0.15 \\
\hline QDs-streptavidin & -15 & $24 \pm 6$ & 0.16 \\
\hline QDs-COOH-PEG & -10.5 & $13 \pm 4$ & \multicolumn{2}{|c|}{0.09} \\
\hline QDs-peptide & - & $46 \pm 22$ & \multicolumn{2}{|c|}{0.04} \\
\hline QDs-Itk-COOH & -41.7 & $49 \pm 19$ & \multicolumn{2}{|c|}{0.025} \\
\hline 25nm Rho-NPs & -14.5 & $5 \pm 3$ & \multicolumn{2}{|c|}{$\begin{array}{l}D_{\text {slow }} \\
0.5\end{array}$} \\
\hline
\end{tabular}

\section{Supplementary Table 3 | Properties and diffusivity of NPs of about 25nm-size}

The diffusivity parameters (immobile fraction and $D_{60 \mathrm{~ms}}$ values) reported in the Table refer to NPs internalized in (untreated) HeLa cells via pinocytosis.

NPs are assumed to be immobile if their MSD curve is smaller than the pointing accuracy of our imaging systems $(\sim 60 \mathrm{~nm})$. Errors on the immobile fraction are SEM.

The number of trajectories used for the calculation of the diffusivity parameters are: $N=1696$ for QDs-PEG-NH $\mathrm{NH}_{2}, N=685$ for QDs-streptavidin, $N=6531$ for QDs-COOH-PEG, $N=785$ for QDs-peptide, $N=915$ for QDs-Itk-COOH, and $N=1314$ for $25 \mathrm{~nm}$ Rho-NPs. 